\title{
3D Biomimetic Tongue-Emulating Surfaces for Tribological Applications
}

\author{
Efren Andablo-Reyes, Michael Bryant, Anne Neville, Paul Hyde, Rik Sarkar, Mathew Francis, \\ and Anwesha Sarkar*
}

Cite This: ACS Appl. Mater. Interfaces 2020, 12, 49371-49385

Read Online

\section{ACCESS | Lلll Metrics \& More | 回 Article Recommendations | st Supporting Information}

ABSTRACT: Oral friction on the tongue surface plays a pivotal role in mechanics of food transport, speech, sensing, and hedonic responses. The highly specialized biophysical features of the human tongue such as micropapillae-dense topology, optimum wettability, and deformability present architectural challenges in designing artificial tongue surfaces, and the absence of such a biomimetic surface impedes the fundamental understanding of tongue-food/fluid interaction. Herein, we fabricate for the first time, a 3D soft biomimetic surface that replicates the topography and wettability of a real human tongue. The 3D-printed fabrication contains a Poisson point process-based (random) papillae distribution and is employed to micromold soft silicone surfaces with wettability modifications. We demonstrate the unprecedented capability of these surfaces to replicate the theoretically defined and simulated collision

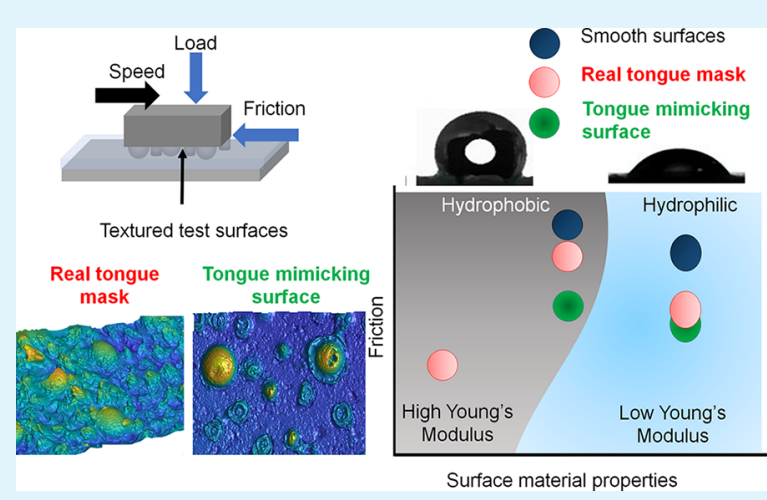
probability of papillae and to closely resemble the tribological performances of human tongue masks. These de novo biomimetic surfaces pave the way for accurate quantification of mechanical interactions in the soft oral mucosa.

KEYWORDS: biomimetic, surface engineering, 3D printing, mesh generation, Poisson point process wettability, soft tribology, lubrication, friction

\section{INTRODUCTION}

Mammalian tongues surfaces are textured with complex geometries, usually at sizes of hundreds of microns. Their high deformability and sophisticated topology, combined with optimum wettability, produce precisely calibrated oral friction and lubrication necessary for controlling highly evolved biophysical activities. Examples include anisotropic hollow papillae on a cat's tongue for effective grooming ${ }^{1}$ or the dynamic erectile papillae in the tongues of nectar-feeding bats and birds enhancing nectar sucking abilities. ${ }^{2}$ In humans, the tongue is integral to elemental activities of food transport, sensory perception of taste and texture, as well as speech. ${ }^{3-5}$ In mechanical terms, the human tongue is a soft tissue containing large asperities better known as papillae. Papillae located in the dorsal anterior section of the tongue, ${ }^{6}$ such as fungiform and filiform papillae are the key players in oral tribology. Put simply, they govern the friction and lubrication at the interface between food/oral fluids and the tongue. Oral tribology has aroused growing interests in fundamental understanding of how exogenously administered fluids, such as food, ${ }^{7,8}$ oral medicines, ${ }^{9}$ oral care products, ${ }^{10}$ and internal fluids such as saliva ${ }^{11}$ interact with the tongue and generate complex textural perception.

Oral tactile sensation, for example, the highly desirable smoothness ${ }^{8}$ of cheese and chocolate to the astringency ${ }^{12}$ in wines, assessed by the human tongue are highly sought after aesthetic properies ${ }^{13}$ that inform food quality, palatability, choice, and ultimately influence the decision of a consumer to ingest a prospective offering. ${ }^{14}$ It is now well-recognized that many complex textural perceptions originate at the oral tribological limit. ${ }^{7,8,12,15}$ Despite its obvious importance, the tribological mechanism remains largely enigmatic because classical rheological approaches typically used for bulk textural quantifications do not provide access to the measurement of the surface-driven lubrication properties. ${ }^{7,8}$ Thus, specialist human tasters are employed in the iterative development cycles of food for oral textural assessment, which makes the process costly, time-intensive and subjective. Despite the high sensitivity of human perception, in vivo assessment does not provide any objective understanding of the underlying physical mechanism behind the success or failure of a product to confer the just-right texture.

Received: July 22, 2020

Accepted: October 12, 2020

Published: October 26, 2020 
Tribometers with metallic surfaces that were originally designed for quantifying friction in high-speed engineering applications are ubiquitously applied to measure oral friction and concurrently do not provide an accurate quantification of oral friction. ${ }^{7-10,15}$ Although advances have been made in recent years in adapting those tribological setups to the oral tribology context, true emulation of the complex anatomical features of a real tongue surface and its physical performance remains incomplete. ${ }^{16-19}$ For instance, silicone surfaces commonly employed in combination with low working loads, typically below $5.0 \mathrm{~N}^{20,21}$ result in contact pressures above $200.0 \mathrm{kPa}^{20}$ which are about 1 order of magnitude higher than the maximum pressure of the human tongue-palate interface $(\sim 50 \mathrm{kPa}) .{ }^{22,23}$ Smooth elastomeric surfaces are generally used as the current state-of-the-art for in vitro oral tribology testing. However, they do not represent the highly textured tongue surfaces in vivo. Although textured surface consisting of regular arrays of less-deformable hydrophobic pillars $^{17}(\sim 2.4 \mathrm{MPa})$ have been used for tribology testing, such surfaces lack the complex random spatial arrangement of biological tissue, and heterogeneity of geometries of the papillae found in natureengineered tongue impedes the biofunctional emulation of tribology. It is thus unclear how, or indeed if the generated measurements during these unrealistic conditions can generate any insights on an in-depth tribological mechanism underlying oral texture.

To sum it all up, creating a surface with relevant properties that mimics the intricate architectural features and, more importantly, the mechanosensing and tribological performance of the human tongue is paramount to gain a quantitative understanding of how fluids interact within the oral cavity. Such frontier knowledge will open up possibilities to replace significant proportions of human sensory studies that are timeconsuming, expensive, and prone to large variations. It will thus enable product developers to perform high-throughput objective screenings of innovative newly designed products and accelerate development process. In addition, a biomimetic tongue could offer myriad applications to quantify adulteration $^{24}$ and counterfeit detection in food and other orally administered high-value fluids whether textural attributes are the governing features and save huge economic loss.

Herein, we present a uniquely fabricated silicone surface that emulates the topology, elasticity and wettability of the human tongue to replicate its oral performance. In order to obtain accurate metrics of the texture of the human tongue, silicone masks of the tongue surfaces in hydrophobic and hydrophilic materials were obtained from healthy human adults. Relevant architectural features of these tongue masks were measured after high quality 3D optical imaging, surface recreation using Screened Poisson reconstruction ${ }^{25}$ including identification of the papillae dimensions, density, distribution, as well as the average roughness of the surfaces. Using these metrics, an innovative biomimetic tongue surface was designed using a Poisson point process-based randomized model ${ }^{26}$ to create a master mold with the appropriate spatial distribution of fungiform and filiform papillae. Additive manufacturing techniques have fuelled the fabrication of high quality replicas of biological organs with intricate geometries and different mechanical properties. ${ }^{27}$ In this study, the mold of the tongue mimicking surface was $3 \mathrm{D}$-printed using advanced digital light processing technology in order to obtain the desired resolution (below $100.0 \mu \mathrm{m}$ ). ${ }^{28}$ Unique 3D biomimetic soft tongue-like surfaces were then obtained by soft lithography of the $3 \mathrm{D}$ - printed artificial surface using soft silicon elastomers with appropriate wettability modification. Besides material properties, we were able to replicate the mechanical performance of a real human tongue for the first time.

A key fundamental question we answered using computational simulation is that how grids, ${ }^{17}$ commonly used in literature to create biological replicas, lack the randomness of real human tongue and consequently fail to replicate the probability of food particle hitting a papillae. We define collision probability per unit distance against a papillae as a novel measure for theoretically quantifying mechanosensing efficiency. We show that this measure differs significantly between grid and random arrangements of papillae. Using computational simulation, we demonstrate for the first time that the randomized arrangement of papillae of precise geometries created in this artificial surface using Poisson point processes closely replicates the predictive mechanosensing of a real human tongue. Tribological experiments using different biopolymers acting as lubricants demonstrated the unprecedented capability of the artificial surfaces formed from the $3 \mathrm{D}$-printed molds to replicate the tribological performance of the polymer surfaces molded on real human tongue masks.

Hence, the 3D human tongue-like surface, i.e., the soft hydrophilic silicone surface textured with randomly distributed papillae-like (both fungiform and filiform) asperities fabricated in this study, represents a new biomimetic approach to quantify oral tribological performance of food and other oral fluids with accuracy. In addition, this offers a unique route forward for predicting mechanosensing and brings a stepchange in our quantitative understanding of oral lubrication. This reduces the necessity for time-consuming and costly human trials consequently reducing the development cycle of oral products. At the same time, experimental and computational insights in this study can be extended to biomimicry of other biological surfaces in the future to match the desired biophysical performance requirement.

\section{EXPERIMENTAL SECTION}

2.1. Materials. For model hydrophilic lubricants, whey protein isolate powder ( $96.3 \mathrm{wt} \%$ protein content) was obtained from Fonterra Limited (Auckland, New Zealand) and xanthan gum with an average molecular weight around $100.0 \mathrm{kDa}$ was purchased from Sigma-Aldrich (Dorset, U.K.) and used without further purification. Dental-grade fast setting Irreversible Hydrocolloid (HS alginate plus) and polyvinyl siloxane impression (Imprint 4 Light Wash) materials were purchased from Henry Schein Dental (Gillingham, U.K.), and they were used for collecting the negative impressions from human participants. Sylgard 184 Silicone (PDMS) elastomer kit was purchased from the Dow Chemical Company (Wiesbaden, Germany) and the silicone monomer and the cross-linking agent were mixed in a 10:1 w/w ratio. Ecoflex 00-30 kit was purchased from Smooth-on Inc. (Pennsylvania, U.S.A.) and the two components were mixed in a 1:1 w/w ratio. The surfactant Span 80 was purchased from Sigma-Aldrich (Dorset, U.K.) and used as received to modify the wettability of Ecoflex $00-30$ by adding the surfactant to the two component mixture at 0.5 wt $\%{ }^{29}$

2.2. Preparation of Lubricants. Whey protein solutions (10.0 wt $\%$ ) were prepared by mixing whey protein isolate powder in $20 \mathrm{mM}$ phosphate buffer at $\mathrm{pH}$ 7.0, prepared using Milli $\mathrm{Q}$ water with a resistivity of $18.0 \mathrm{M} \Omega \mathrm{cm}$ (Milli-Q apparatus, Millipore Corp., (Massachusetts, U.S.A.) and allowed to stir for $2 \mathrm{~h}$ at room temperature to ensure complete dissolution. ${ }^{21}$ Xanthan gum solution (1.0 wt \%) was prepared by mixing the xanthan gum powder in 20 $\mathrm{mM}$ phosphate buffer $(\mathrm{pH} 7.0)$ by stirring for $24 \mathrm{~h}$ at room 
Table 1. Characterization of the Topographic Features of Human Tongue Surfaces ${ }^{a}$

\begin{tabular}{|c|c|c|c|c|c|c|}
\hline & \multirow{2}{*}{\multicolumn{2}{|c|}{ papillae density (per $10^{-4} \mathrm{~m}^{2}$ ) }} & \multicolumn{4}{|c|}{ papillae dimensions } \\
\hline & & & \multicolumn{2}{|c|}{ fungiform } & \multicolumn{2}{|c|}{ filiform } \\
\hline & fungiform & filliform & diameter $(\mu \mathrm{m})$ & height $(\mu \mathrm{m})$ & diameter $(\mu \mathrm{m})$ & height $(\mu \mathrm{m})$ \\
\hline mean & 13.5 & 160 & 878.0 & 390.0 & 355.0 & 195.0 \\
\hline deviation & 1.5 & 30 & 97.0 & 72.0 & 40.0 & 6.5 \\
\hline
\end{tabular}

${ }^{a}$ Average size and density of fungiform and filiform papillae observed in polyvinyl siloxane tongue impressions. The analysis was performed in the middle section of the tongue.

temperature, followed by stirring at $65 \mathrm{rpm}$ in a water bath at $50{ }^{\circ} \mathrm{C}$ for $4 \mathrm{~h}$ in order to ensure optimum solvation of the biopolymer. ${ }^{21}$

2.3. Collection of Human Tongue Silicone Impressions. Negative impressions of the upper surface of the tongue were collected from healthy adults ( $n=15$ subjects, 9 females 6 males, age ranging $18-55$ years, mean age $29.1 \pm 3.7$ years) using two different polymeric materials commonly used for collecting dental impressions, i.e., hydrophobic (polyvinylsiloxane) and hydrophilic (alginate) materials using an appropriately controlled dental procedure (Ethics DREC ref: 120318/AS/245, University of Leeds). Both of the polymers used have a short setting period of about 120 s. Impressions were collected at the Dental Research facilities of the School of Dentistry, University of Leeds. A thin film of approximately $3.0 \mathrm{~mm}$ of either polyvinylsiloxane or alginate was carefully deposited on the anterior upper tongue surface of each participant. The polymer was allowed to set avoiding any movement of the tongue to obtain a reliable reproduction of the tongue surface (negative). The pressure imposed on the tongue during the mask collection corresponds to the weight of the impression material film, which is within 35.0 to $50.0 \mathrm{~Pa}$ given the materials densities are within 1200 and $1700 \mathrm{~kg} \mathrm{~m}^{-3}$. Impressions were carefully disinfected using a 1.0 wt \% Virkon (Lanxess, Cologne, Germany) solution before storing for optical characterization and for soft-lithography experiments. Alginate impressions were kept wrapped in a humid tissue in order to avoid shrinking and were measured within $48 \mathrm{~h}$ following collection.

2.4. Collection of Pig Tongue and Preparation. Tongues from freshly sacrificed pigs (about 6 months old) were processed within $4 \mathrm{~h}$ of collection from a local abattoir. The tissue was washed thoroughly using $20 \mathrm{mM}$ phosphate buffer at $\mathrm{pH} 7.0$ to remove any blood, food, and saliva residue and dried by air blowing. Cylindrical pieces of 1.0 $\mathrm{cm}$ diameter $\times 1.0 \mathrm{~cm}$ height were cut from the tongues using a punching tool to be used for compression testing and laser scanning. Thin (about $2.0 \mathrm{~mm}$ thick) pieces of tissue of the dorsal anterior tongue were carefully dissected using a scalpel for wettability (contact angle) measurement.

2.5. 3D Optical Scanning and Profilometry. 3D laser scanning of animal tissue (pig tongue) was performed using an Artec Space Spider laser scanner (Artec3D, Luxembourg, Luxembourg). The 3D optical analyses of the negative impressions of the human tongues on polyvinylsilaxane and alginate as well as positive impressions obtained using polydimethylsiloxane (PDMS) and Ecoflex 00-30 were performed using an Alicona InfiniteFocus (IF) instrument equipped with $5 \times$ objective. Brightness and contrast were chosen to obtain the best results for each surface scanned. The $3 \mathrm{D}$ space data points were scanned at a resolution of $0.1 \mu \mathrm{m}$ for vertical (z-axis) and $2.0 \mu \mathrm{m}$ for $x-y$ plane. The Alicona IF-MeasureSuite 5.3.4 (OPTIMAX Imaging Inspection \& Measurement Ltd., Leicestershire, U.K.) was used to process the $3 \mathrm{D}$ data set and to obtain average roughness parameters and profile details at microscopic level, i.e., papillae after surface reconstruction as discussed in the next section. Due to the complicated shape of the tongue, an eighth order polynomial algorithm was employed to fit and subtract the underlying curvature of the surfaces while preserving the surface roughness. After applying the form-removal algorithm, it was possible to define a plane on the flat surface. Surface texture was then calculated complying with the International Standard ISO 4287 in a $5.0 \times 5.0 \mathrm{~mm}$ area.

2.6. Surface Reconstruction. The point set obtained from the 3D optical scanning of human tongue masks using the Alicona IF instrument corresponds to a discrete sample $S$ of a continuous surface. The reconstruction of the original continuous surface was performed using a variant of the Poisson surface reconstruction algorithm proposed by Kazhdan et al., ${ }^{30}$ which assumes that the surface is obtained from the boundary (written as $\partial M$ ) of a solid object $M$ (e.g., the tongue in this case). A scalar function $\chi: R^{3} \rightarrow R$, is used to represent $M$, so that $\chi$ is identically 1 in the interior of $M$, and 0 outside of it. For such a function $\chi$, the gradient $\nabla \chi$ is zero everywhere inside and outside of $M$, but is nonzero precisely at the boundary.

The strategy in Kazhdan et al., ${ }^{30}$ is to reconstruct $\chi$ from a given boundary gradient vector field $\vec{V}: \mathbb{R}^{3} \rightarrow \mathbb{R}^{3}$. The best reconstruction can be seen as a minimization of a function $\mathrm{E}$ defined in eq 1 :

$$
E(\chi)=\int\|\nabla \chi-\vec{V}(p)\|^{2} \mathrm{~d} p
$$

This minimization of $E$ is equivalent to finding a solution for $\chi$ that provides the best fit of the gradient field to $\bar{V}$, and can be shown to be equivalent to solving a Poisson problem: $\Delta \chi=\nabla \cdot \bar{V}$. Infinite magnitudes on the gradient vector field can be avoided using a Gaussian smoothing of $\chi$. A set of vectors defined on the sample set $S$ forms a sampling of a gradient vector field.

Computationally, the $3 \mathrm{D}$ optical scan data $S$ is mapped to an Octree, which is an iterative decomposition of the $3 \mathrm{D}$ space into successively smaller cubes containing the sample points. The depth $d$ of the Octree, supplied as a parameter, sets the size of the smallest cubes to $2^{-d}$ times the diameter of the point set. Gradients at the cubes of the Octree are computed using interpolation of samples in neighboring cubes. The boundary $\partial M$ is obtained as an iso-surface of $\chi$.

Meshlab $^{a}$ was used for reconstructing the surfaces, and these are visualized as smoothened surfaces and triangulated meshes and were used to quantify the average size and density of the papillae. Meshlab ${ }^{a}$ implements a more refined technique called Screened Poisson surface reconstruction, ${ }^{25}$ which forces the surface to be close to the original sample points by penalising for deviation from samples. The papillae distribution and sizes of filiform and fungiform were calculated in an approximately $2.0 \times 2.0 \mathrm{~cm}$ area populating the mid sections of each tongue and approximately $0.5 \mathrm{~cm}$ from the tip of the tongue. Values obtained from the analysis are presented in Table 1.

2.7. Surface Generation and 3D Printing. To create the 3D biomimetic tongue-like surface, we simulated the positions of filiform and fungiform papillae based on a spatial Poisson point processes. A similar approach has been used to model the distribution of biological features such as neurons and synaptic junctions in rat brains. ${ }^{26}$ Briefly, a simple spatial Poisson point process will place each point independently on a plane, by randomly selecting a value for the $\mathrm{x}$ coordinate and another random value for the $y$ coordinate, each chosen from a uniform distribution. On any given region of the domain, such a selection of points is known to follow the Poisson distribution. If $X$ is a random variable denoting the number of points in a unit area, then its probability distribution is given by the Poisson distribution in eq 2 :

$$
\operatorname{Pr}(X=n)=\frac{\lambda^{n} \mathrm{e}^{-\lambda}}{n !}
$$

where $\lambda$ is called the rate of the distribution and is the expected number of points in a unit area. 
We generated papillae positions on a $1.0 \mathrm{~cm} \times 1.0 \mathrm{~cm}$ region using spatial Poisson point processes (by selecting random $x$ and $y$ coordinates in the region) with rates corresponding to papillae density. The fungiform papillae were first generated one by one as a Poisson process (see Table 1 for papillae density). If the location of a new fungiform papilla overlapped with one of the already placed papillae then the new one was discarded and the attempt repeated until a nonoverlapping location was selected. Next, the filliform papillae were generated in a similar fashion, checking for overlaps with all previous fungiform and filliform papillae to create the final model surface for $3 \mathrm{D}$ printing. This model surface was produced using AutoCAD 2018 (Autodesk, Inc., London U.K.) with a resolution in the order of hundreds of microns mimicking both fungiform and filiform papillae.

The AutoCAD tongue-like surface models were printed using a Perfactory P3 mini 3D printer model (EnvisionTEC, Dearborn, U.S.A.), which utilizes digital light processing technology. The high temperature molding material used for impressions (HTM140) inhibited the cross-linking process of polydimethylsiloxane and Ecoflex 00-30 and thus hindered the soft-lithography process (replica-molding) later on. In order to obtain a proper finish on the molded surfaces, the surface of the mold was covered by a thin film of poly(vinyl alcohol) (PVA). The PVA film was obtained by depositing a thin liquid film of $0.3 \mathrm{wt} \%$ PVA solution on top of the 3D printed surface, followed by evaporation at $50{ }^{\circ} \mathrm{C}$.

2.8. Soft-Lithography. Silicone surfaces mimicking tongue properties (topographic feature, deformability, and wettability) were created for tribological studies by replica molding in different siliconbased polymers as shown in Table 2. Polyvinyl siloxane negative

Table 2. Silicon Surfaces Generated Including the 3DPrinted Biomimetic Tongue-Like Surface ${ }^{a}$

\begin{tabular}{llcl}
$\begin{array}{c}\text { surface } \\
\text { nomenclature }\end{array}$ & \multicolumn{1}{c}{ polymer } & $\begin{array}{c}\text { span } 80 \\
(\text { wt } \%)\end{array}$ & \multicolumn{1}{c}{ mold } \\
\hline $\begin{array}{l}\text { PDMStongue } \\
\text { Ecohbtongue }\end{array}$ & PDMS & 0.0 & tongue mask \\
Ecohltongue & Ecoflex 00-30 & 0.5 & tongue mask \\
Ecohbprint & Ecoflex 00-30 & 0.0 & 3D printed master \\
Ecohlprint ${ }^{b}$ & Ecoflex 00-30 & 0.5 & 3D printed master \\
PDMS smooth & PDMS & 0.0 & $\begin{array}{c}\text { smooth steel surface } \\
\left(R_{\mathrm{a}}=50 \mathrm{~nm}\right) \\
\text { Ecohb smooth } \\
\text { Ecoth steel surface } \\
\left(R_{\mathrm{a}}=50 \mathrm{~nm}\right)\end{array}$ \\
& Ecoflex 00-30 & 0.0 & $\begin{array}{c}\text { smooth steel surface } \\
\left(R_{\mathrm{a}}=50 \mathrm{~nm}\right)\end{array}$
\end{tabular}

${ }^{a}$ Nomenclature of the surfaces made of different polymers and molded on either a polyvinylsiloxane real human tongue mask or 3Dprinted microstructured master for the tribological tests. Smooth surfaces are controls cast using steel surfaces with $50 \mathrm{~nm}$ surface roughness. Span 80 has been added in some surfaces for wettability modification. ${ }^{b}$ Represents the final biomimetic tongue-like surface emulating the topological features, deformability and wettability of a human tongue.

impression of a human tongue as having an average density of fungiform and filiform papillae was identified, this was used as a master mold to create positive impressions for the tribological studies. Surfaces were cast in three materials, PDMS, Ecoflex 00-30 and Ecoflex 00-30 containing 0.5 wt \% Span 80 .

For the biomimetic tongue surfaces, a 3D-printed mold was used as the master for the replica-molding process. In this case, surfaces were made of either Ecoflex 00-30 or Ecoflex 00-30 containing 0.5 wt \% Span 80 . Molding procedures varied slightly from PDMS to Ecoflex 00-30. Kits for both materials are composed of two components. After mixing the two components at the appropriate ratio, homogeneous mixtures were achieved using a Thinky Planetary mixer and degassing system ARE-250, Intertronics (Kidlington, U.K.) with a mixing cycle of $2 \mathrm{~min}$ at $2000 \mathrm{rpm}$, followed by $1 \mathrm{~min}$ degassing at $2200 \mathrm{rpm}$.
Immediately after mixing, either PDMS or Ecoflex 00-30 was carefully poured onto the master surface (negative human tongue impressions or 3D-printed negative masters) avoiding the formation of bubbles. Cross-linking of PDMS was achieved by placing the mold at $60{ }^{\circ} \mathrm{C}$ for $4 \mathrm{~h}$. Ecoflex 00-30 was cured for $5 \mathrm{~h}$ at room temperature.

2.9. Theoretical Analysis of Collision Probability and Its Computational Simulation. We introduce collision probability per unit distance as a measure of mechanosensing efficiency of an arrangement of papillae. It is the probability that a unit movement of a particle on the surface of the tongue will collide with one or more papillae. Mechanosensing in this case can be defined as the ability of the papillae to sense any mechanical cues of the food particles in the oral environment.

To evaluate this quantity, let us imagine a small area of the tongue to be represented by a plane and consider papillae of radius $r$ arranged in a given arrangement. Our interests are in grid arrangements and spatial Poisson distribution (defined in section 2.7), where the location of a papilla is selected randomly on the area, so that any unit area is equally likely to contain the papilla.

The computational evaluation of measure for grid and Poisson distributions was done on a $2 \mathrm{D}$ plane model with papillae represented as disks with varying density. For each density value, 20 Poisson distribution arrangements with suitable density were created over an area of the plane and grids of same area and same density were generated. On each such arrangement, 100 random straight segments were generated and tested for collision with papillae. Thus, each data point correspond to 2000 trials. For comparison with real tongue, we took a section of real tongue impression from our database and recorded the locations of filiform and fungiform papillae on it. This was compared with a plane with both filiform and fungiform papillae at their mean densities (Table 1) for grid and Poisson distributions.

2.10. Rheology and Adsorption Behavior Model Lubricants. All rheological characterization was performed using a Kinexus Ultra+ rotational rheometer (Malvern Instruments, Malvern U.K.), equipped with a $60.0 \mathrm{~mm}$ diameter stainless steel cone-on-plate geometry. Measurements were performed at fixed temperature of $37^{\circ} \mathrm{C}$. Steady shear viscosity measurements were performed in shear control mode covering a range from 1.0 to $1000.0 \mathrm{~s}^{-1}$. For each point, steady state was achieved within a tolerance of $0.5 \%$. Three measurements were conducted for each sample and results are presented as means and standard deviations of triplicate samples $(n=3 \times 3)$.

The adsorbed hydrated mass of the model lubricants on PDMScoated sensors were measured using a quartz crystal microbalance with dissipation monitoring (QCM-D) (E4 system, Q-Sense, Gothenburg, Sweden) as described previously. ${ }^{1,31-33}$ For the preparation of PDMS-coated QCM-D sensors, $10 \mathrm{wt} \%$ PDMS in toluene solution was prepared and left to stir for $24 \mathrm{~h}$. Then the solution was further diluted with toluene to $0.5 \mathrm{wt} \%$ which was again left to stir for $24 \mathrm{~h}$. Silica-coated QCM-D sensors (QSX-303, Q-Sense, Gothenburg, Sweden) were immersed in RCA silicon wafer cleaning solution ( 5 parts of deionized water, 1 part of ammonia and 1 part of aqueous $\mathrm{H}_{2} \mathrm{O}_{2}$ (hydrogen peroxide, $30 \%$ )) at $80{ }^{\circ} \mathrm{C}$ for $15 \mathrm{~min}$ to remove any organic material and insoluble particles, followed by three cycles of sonication in ultrapure water for $10 \mathrm{~min}$ each cycle before drying using liquid nitrogen gas. Finally, $100 \mu \mathrm{L}$ of $0.5 \mathrm{wt} \%$ PDMS solution was placed on the substrate and was spin-coated at $5000 \mathrm{rpm}$ speed for $60 \mathrm{s.}^{34}$

The hydrophobic PDMS-coated sensors were cleaned by $30 \mathrm{~s}$ immersion in toluene, followed by $30 \mathrm{~s}$ immersion in isopropanol, then $2 \mathrm{~min}$ immersion in ultrapure water, drying with nitrogen gas and letting the remaining solvent molecules evaporate for $2 \mathrm{~h}$. The whey protein or xanthan gum were supplied into the QCM-D chamber containing the PDMS-coated sensors by a peristaltic pump with a flow rate of $100 \mu \mathrm{L} / \mathrm{min}$ at $25{ }^{\circ} \mathrm{C}$. The first step was to inject the buffer solution until a stable baseline was observed. Subsequently, for the adsorption of whey protein or xanthan gum solution on PDMS surfaces, solutions were injected into the system for at least an hour, allowing the system to equilibrate, followed by rinsing in buffer solution for $30 \mathrm{~min}$. The data were fitted using the Voigt model for viscoelastic solids (namely, "Smartfit Model") by Dfind software (Q- 
Sense, Gothenburg, Sweden) to obtain the mass of the hydrated layers. Three measurements were conducted for samples in triplicates and means and standard deviations were reported $(n=3 \times 3)$.

2.11. Tribology in the Presence of Model Lubricants. Tribological studies were carried out using a Kinexus Ultra+ rotational rheometer (Malvern Instruments, Malvern U.K.), equipped with a $50.0 \mathrm{~mm}$ diameter stainless steel plate-on-plate geometry. Silicone surfaces created using soft lithography for both the negative impressions of human tongue surfaces and 3D-printed masters measuring $2.0 \mathrm{~cm} \times 2.0 \mathrm{~cm}$ (Table 2) were glued at the rim of the top plate. The tribological contact was then formed by the silicon surface against a stainless steel plate. Experiments were performed in normal force $\left(F_{\mathrm{N}}\right)$ control and shear rate control mode. Normal force was fixed for all experiments at $1.0 \mathrm{~N}$. Shear rate was chosen in order to sweep angular speeds ranging from 0.005 to $1.0 \mathrm{~s}^{-1}$. New silicone surfaces were used whenever the lubricant was changed. For each velocity, torque $(M)$ was recorded for at least two rotations of the upper geometry to ensure an optimum ensemble average. Friction coefficient $\mu$ was calculated using torque values following eq 3 .

$$
\mu=\frac{M}{R F_{\mathrm{N}}}
$$

where $R$ stands for the plate radius $(R=0.025 \mathrm{~m})$. Friction coefficient $(\mu)$ is presented as a function of the linear speed $V_{\mathrm{R}}$ at the rim of the plate and is calculated by $V_{\mathrm{R}}=\Omega R$, where $\Omega$ is the angular speed. The contact was flooded to cover a height of $0.3 \mathrm{~cm}$ with respect to the lower baseline of the top surface. Tribological experiments were also performed using a Mini Traction Machine (MTM2, PCS Instruments, London U.K.) equipped with a smooth hydrophobic PDMS (2.4 $\mathrm{MPa})$ ball (19.0 mm diameter) on disk contact to serve as a control with entrainment speed $U$ ranging from 0.3 to $0.005 \mathrm{~m} / \mathrm{s}$ and slidingrolling ratio at $50.0 \%$. The load was fixed at $2 \mathrm{~N}$, resulting in a Hertz contact pressure of $\sim 200.0 \mathrm{kPa}^{7,11}$ and contact radius of $1.0 \mathrm{~mm}$. Experimental temperature was fixed at $37{ }^{\circ} \mathrm{C}$. Three measurements were conducted for each sample and results are presented as means and standard deviations of triplicate samples $(n=3 \times 3)$.

2.12. Young's Modulus and Wettability of Surfaces. Mechanical compression tests were performed on the surfaces in Table 2 and pig tongue surfaces using a TA-TX2 texture analyzer (Stable Micro Systems Ltd., Surrey, U.K.) equipped with a flat $1.0 \mathrm{~cm}$ diameter geometry. Compression was performed at a constant speed of $0.002 \mathrm{~m} \mathrm{~s}^{-1}$ to a maximum strain of $40.0 \%$. Tests were performed at a fixed temperature of $25^{\circ} \mathrm{C}$. The linear region at low true strain of 0.05 was fitted in order to calculate Young's modulus (linear slope) for the materials.

Static water contact angle measurements were performed on the surfaces in Table 2 and pig tongue surfaces by means of sessile drop technique using an OCA25 drop tensiometer (DataPhysics Instruments, Filderstadt, Germany). For contact angle measurements a water drop of $5 \mathrm{~mL}$ was deposited on the surface and the drop profile was analyzed using the SCA 20 software (DataPhysics Instruments, Filderstadt, Germany) to determine the contact angle at the water/ solid/air interface.

2.13. Scanning Electron Microscopy. Scanning electron microscopy images were captured for the silicone tongue mimicking surfaces and pig's tongue using EVO MA15 scanning electron microscope (Carl Zeiss, Jena Germany). In order to facilitate imaging, surfaces were mounted on $12.0 \mathrm{~mm}$ diameter stubs and were coated with a thin gold layer $(\sim 5 \mathrm{~nm})$ by sputtering.

2.14. Theoretical Approximation to Surface Deformation. In the case of the biomimetic tongue surface, pressure can be calculated by considering papillae as semi spherical shapes and filiform papillae as cylinders. Thus, the total force in the contact $F_{\mathrm{N}}$ is distributed between the two different species. On the one hand, fungiform papillae can be approximated as a semi spherical shape and its deformation can be quantified using the Hertz contact theory. ${ }^{35}$ In the case of finite deformation, the indentation $(\delta)$ and contact radius $\left(a_{H}\right)$ of a ball-on-plate contact are given by eqs $4-6$ :

$$
\delta=\frac{a_{\mathrm{H}}^{2}}{R}-f\left(\frac{a_{\mathrm{H}}}{R}\right) \frac{W_{\mathrm{S}}}{\pi R E\left(1-\nu^{2}\right)}
$$

and

$$
a_{\mathrm{H}}^{3}=\frac{3}{4} \frac{W_{\mathrm{S}} R}{E}
$$

with

$$
f\left(\frac{a_{\mathrm{H}}}{R}\right)=\frac{2(1+v)}{\left(4+\left(\frac{a_{\mathrm{H}}}{R}\right)^{2}\right)^{3 / 2}}+\frac{\left(1-v^{2}\right)}{\left(4+\left(\frac{a_{\mathrm{H}}}{R}\right)^{2}\right)^{1 / 2}}
$$

Here $W_{\mathrm{S}}$ is the load supported by each fungiform papillae, $E$ is the Young's modulus, $R$ is the papillae radius, and $\nu$ is the Poisson's ratio. On the other hand, filiform papillae are approximated as cylinders of length $L_{0}$ and radius $a$. Thus, for a Hencky strain $\varepsilon=-\ln \left(\left(L_{0}-L\right) /\right.$ $L_{0}$ ), with $L$ as the deformed length of the cylinder, the force $W_{\mathrm{C}}$ in the cylinder is given by eq $7: 36$

$$
W_{\mathrm{C}}=\pi a^{2} E \varepsilon L_{0} /\left(L_{0}-L\right)
$$

Then the total $W_{\mathrm{T}}$ force supported by fungiform and filiform papillae is given by eq 8 :

$$
W_{\mathrm{T}}=N_{\mathrm{S}} W_{\mathrm{S}}+\Theta(R-\delta) N_{\mathrm{C}} W_{\mathrm{C}}
$$

where $N_{\mathrm{S}}$ and $N_{\mathrm{C}}$ are the number of fungiform and filiform papillae in the contact, respectively, and $\Theta(R-\delta)$ is a step function defined as

$$
\Theta(R-\delta)=\left\{\begin{array}{l}
0.0, R-\delta>L_{0} \\
1.0, R-\delta<L_{0}
\end{array}\right.
$$

Average pressure in fungiform papillae was calculated as $1.5 W_{\mathrm{S}} /\left(\pi a_{\mathrm{H}}{ }^{2}\right)$ and in filiform papillae it was $W_{\mathrm{C}}\left(L_{0}-L\right) /\left(\pi a^{2} L_{0}\right)$.

\section{RESULTS AND DISCUSSION}

We aimed to create a biomimetic surface that fulfills the following requirements: (1) The synthetic tongue surface should mimic the intricate topography of human tongue with precise geometry and density of the filiform and fungiform papillae per unit area. Here, the spatial arrangement of papillae is paramount to replicate mechanosensing accurately. (2) The surface has to be mechanically compliant to generate pressures that closely resemble the real oral pressures, which is crucial for tribological analysis. (3) The silicon surface should possess appropriate hydrophilicity emulating that of a real tongue. Finally, (4) all of these architectural features should endow the newly designed biomimetic surface with frictional properties akin to the way a real tongue mask behaves in a tribological setup.

3.1. Biomimicry of the Topographic Features of Human Tongue. To emulate the surface geometry of the architecturally complex human tongue, it was imperative to first systematically characterize the dorsal tongue surfaces. Tongue masks were collected using hydrophobic and hydrophilic polymeric materials from 15 healthy participants (Ethics DREC ref: 120318/AS/245, University of Leeds). Figure 1a,b shows negatives of the $3 \mathrm{D}$ optical scans of masks of a real human tongue belonging to the same participant produced using hydrophobic (polyvinyl siloxane) and hydrophilic (alginate) polymers, respectively.

Despite the difference in wettability of these two masking polymers, the features presented in optical scans are identical. Both masks show the presence of clear circular holes produced 

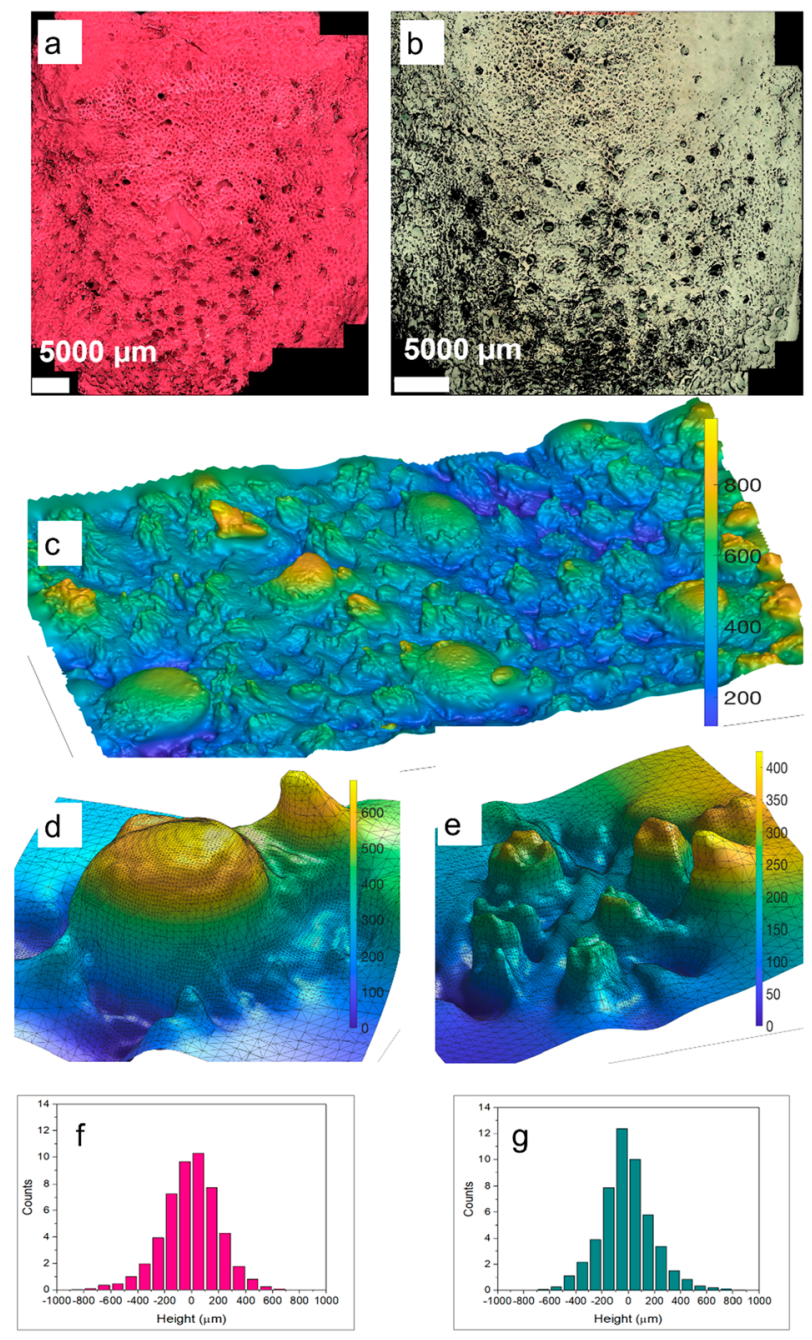

Figure 1. Characterization of human tongue surfaces using polymeric impressions. Negative 3D optical scans of the tongue impressions taken using (a) hydrophobic (polyvinyl siloxane) and (b) hydrophilic (alginate) masking materials where the papillae are visible as circular holes. (c) Positive 3D optical image using polydimethylsiloxane (PDMS) mask of the human tongue obtained using the hydrophobic impression shown in panel a. (d) Soft-lithography technique showing zoomed image of fungiform papillae with their characteristic dome shape (e) filiform papillae as crown-shaped aggregate of pillars. The meshes of tesselated triangles in panels $\mathrm{d}$ and e are generated using Screened Poisson surface reconstruction of the point data sets obtained from the $3 \mathrm{D}$ optical scanning conforming accurately to the surfaces of $3 \mathrm{D}$ objects. Distribution of heights are shown for (f) hydrophobic and $(\mathrm{g})$ hydrophilic impressions.

by fungiform and filiform papillae present in the anterior dorsal section of the human tongue.

To clearly understand the papillae distribution, Figure 1c shows the optical scan of a positive mask of the same tongue obtained in a polydimethylsiloxane (PDMStongue) cast from the hydrophobic negative mask shown in Figure 1a. In Figure 1c, one can clearly appreciate the roughness of the tongue surface caused by the microstructured surface with presence of both filiform and fungiform papillae, clearly distinguishable by their well-differentiated shapes. Figure 1d,e shows meshes of the tesselated triangles fitting tightly to the surfaces of the real $3 \mathrm{D}$ tongue surface that are rendered from scanned point data sets (using eq 1 in the Method section). On one hand, Figure
$1 \mathrm{~d}$ shows that the fungiform papillae have dome or mushroomshapes surrounded by numerous filiform papillae.

On the other hand, Figure 1e shows that the filiform papillae are clusters of cylinders with irregular crown-like ends and are significantly thinner in comparison to the fungiform papillae (Figure 1d).

Figure 1f,g shows the height distribution of both hydrophobic and hydrophilic surfaces covering the whole area shown in Figure 1a,b, respectively. Both the polymeric materials used for masking demonstrate similar height distributions, with alginate having a slightly narrower distribution in comparison to polyvinyl siloxane. Supplementary Table $S 1$ shows average principal values of the surface texture, $S_{a}, S_{q}, S_{\mathrm{p}}$ and $S_{\mathrm{v}}$ for six different regions of the anterior tongue surface from the tip up to $2.0 \mathrm{~cm}$ from the back. Values of average height $S_{\mathrm{a}}$ vary from 119.0 to $142.0 \mu \mathrm{m}$ for impressions in polyvinyl siloxane and from 83.0 to $118.0 \mu \mathrm{m}$ for alginate. The root-mean-square height $S_{\mathrm{q}}$ was found to range from 142.0 to $174.0 \mu \mathrm{m}$ for impressions in polyvinyl siloxane and from 105.0 to $150.0 \mu \mathrm{m}$ for alginate. Average surface roughness parameters $S_{\text {a }}$ and $S_{\mathrm{a}}$ were slightly higher in the case of the hydrophobic material (polyvinyl siloxane) in comparison to the hydrophilic material (alginate). Values of $S_{\mathrm{a}}$ and $S_{\mathrm{q}}$ reported here are larger but in the same order of magnitude of others reported elsewhere, ${ }^{16}$ where impressions were collected in a different manner likely involving a larger pressure during the mask acquisition as compared to the current study.

It is noteworthy that the surface roughness parameter alone (Supplementary Table S1) is not sufficient to achieve such higher order biomimicry as needed in case of a tongue with the topological complexity and geometric variations as observed in Figure 1c. Due to similarities among impressions taken on alginate and polyvinyl siloxane, the analysis is shown only for the latter in Table 1. Preference on polyvinyl siloxane over alginate impression was due to the fact that alginate tends to show considerable shrinkage after $48 \mathrm{~h}$ storage and was not appropriate to create positive surfaces for further studies. Fungiform papillae are, on average, approximately twice as wide and tall as the individual filiform papillae.

Apart from the fungiform height, which presents about $20.0 \%$ of interindividual variation, the rest of the papillae dimensions have less than $11.0 \%$ deviation in mean size values. Number density of filiform is at least 1 order of magnitude larger than fungiform. As might be expected, a large deviation of about $20.0 \%$ from the average density values in the case of the filiform distribution was observed, indicating the interindividual variability. However, the distribution of fungiform papillae shows a relatively smaller deviation of less than $12.0 \%$. We now created the automatic computer-aided design (AutoCAD) of the tongue mimicking surface using Poisson point process-based randomization (using eq 2 in the method section) for 3D printing using the heterogeneous geometry of filiform and fungiform papillae including their exact dimensions and density as shown in Table 1 . Table 2 presents a summary of the different surfaces produced by replicamolding on the $3 \mathrm{D}$ printed microstructured master as well as on a human tongue mask (Figure 1c) (see methods for fabrication details).

Surfaces also included hydrophilic ones where wettability modification was introduced by addition of surfactant, i.e., Span $80^{29}$ (Table 2) during the cross-linking process of the polymer after the replica-molding against the 3D-printed master. 

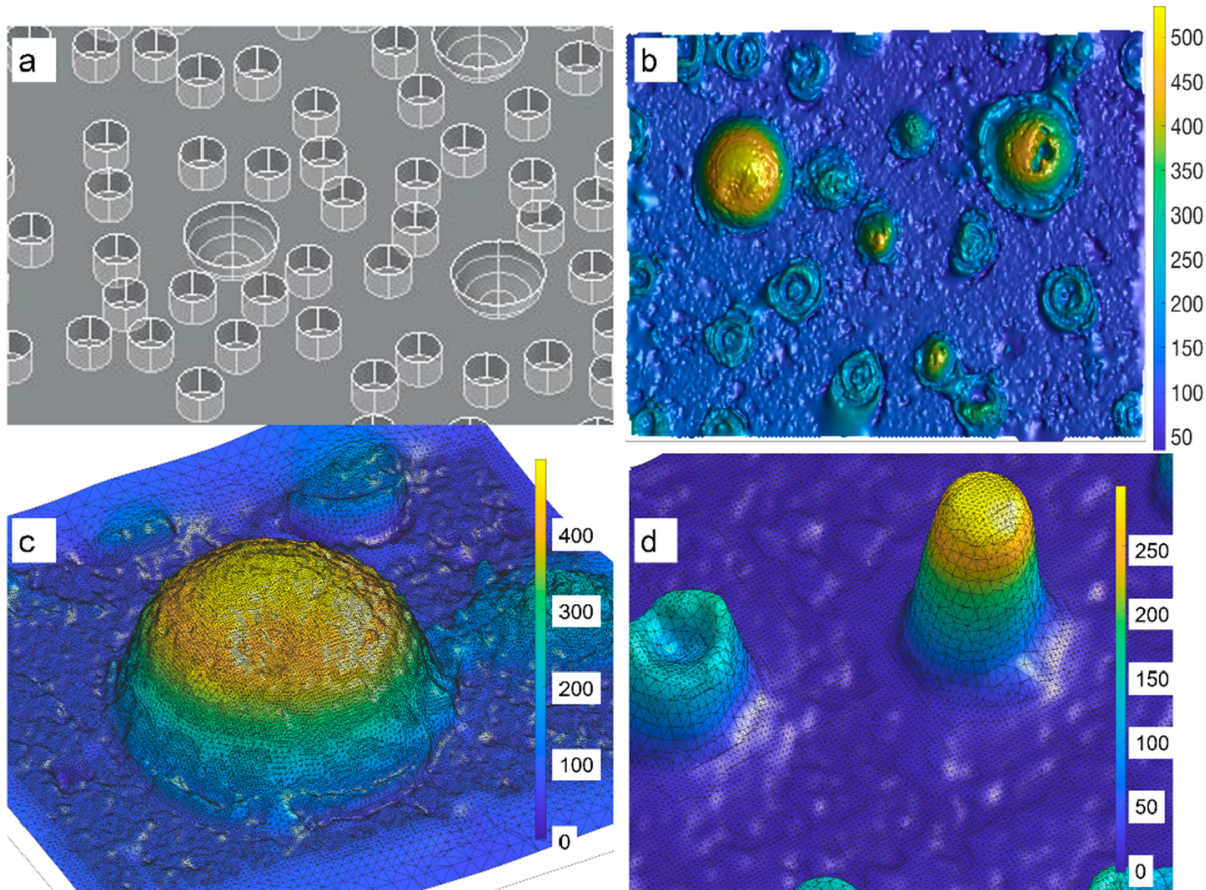

Figure 2. Topographic design of the biomimetic tongue-like surface. (a) Topographic design generated using Poisson point process with random arrangement of dome shapes and cylinders, mimicking the fungiform and filiform papilla respectively, for 3D-printing using digital light processing technology. (b) Positive impressions of the 3D optical scan of the biomimetic tongue-like surface cast in soft Ecoflex 00-30 using soft-lithographic process of 3D-printed mold presented in figure (a). Triangulated meshes of single (c) fungiform and (d) filiform papillae within the soft Ecoflex 0030 biomimetic tongue-like surface is generated using Screened Poisson surface reconstruction of the point data sets obtained from the 3D optical scanning.
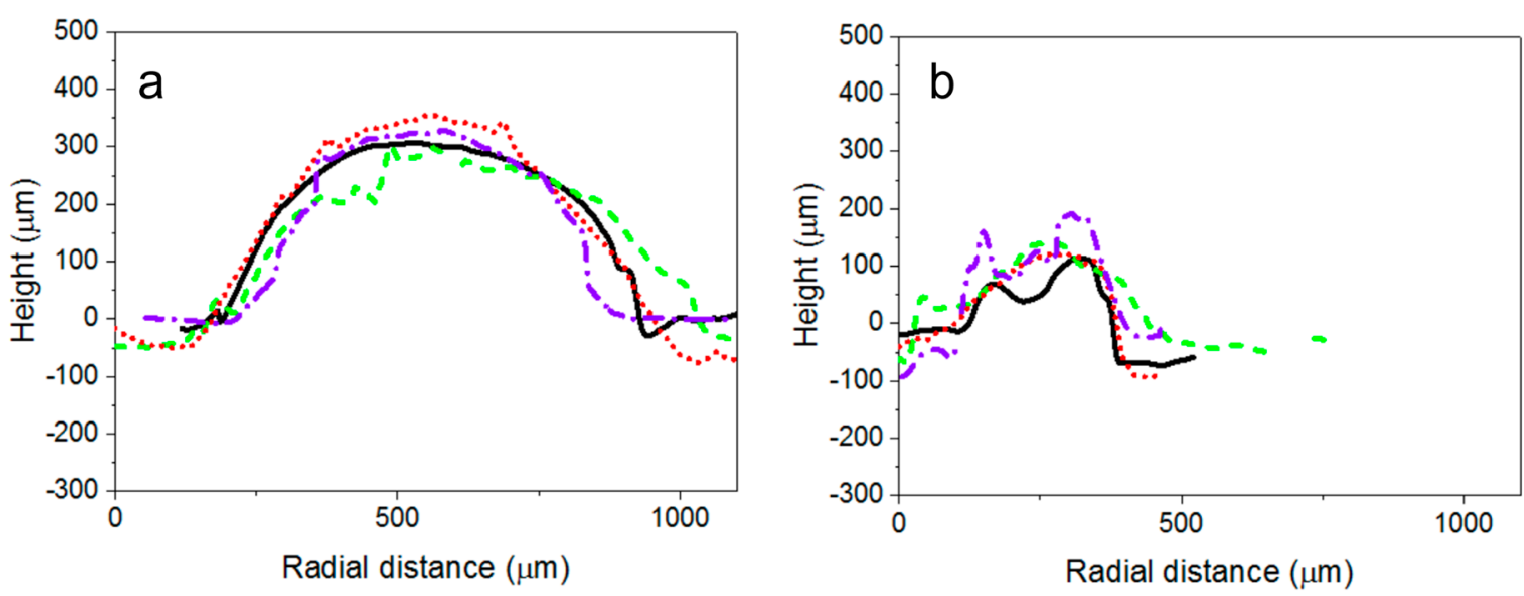

Figure 3. Topographic comparison of replica molded surfaces of tongue masks and 3D-printed tongue-like polymeric surfaces. Topographic height of (a) fungiform and (b) filiform papilla obtained from triangular meshes of the point data sets obtained from the 3D optical scanning of casts of human tongue impressions using PDMS, i.e., PDMStongue (black continuous line), Ecoflex 00-30 i.e. Ecohbtongue (green dashed line) and Ecohltongue (purple dashed line) and cast of a 3D-printed master using Ecoflex 00-30, i.e., Ecohbprint (red dotted line).

To confirm whether the biomimicry obtained using 3Dprinting and replica-molding has achieved high fidelity and definition (see the AutoCAD design in Figure 2a), a top view of a $3 \mathrm{D}$ optical scan of the silicone surface Ecohbprint, i.e., the surface created from the 3D printed master using Ecoflex 0030 as the polymeric material by replica-molding process is shown in Figure $2 b$.

The scanned image shows the random distribution of papillae across the surface with filiform papillae having a larger density in comparison to the fungiform papillae. The domeand the cylindrical-shaped fungiform and filiform papillae can be observed in Figure 2c,d, respectively, on the $3 \mathrm{D}$ printed surface. It is worth noticing the remarkable similarity in the design features between papillae presented in Figure 2c,d on the $3 \mathrm{D}$ printed surface generated using randomized distribution of the surface features with those in the real human tongue surface shown in Figure 1d,e, (fungiform and filiform, respectively). The solid body of fungiform and filiform papillae as shown in Figures 1d,e and 2c,d, are approximated as domelike and cylindrical-like shapes, respectively. Thus, on average, papillae will be assumed as radially symmetric solid bodies for the following calculations. To validate whether the engineered 
surfaces mimic the intricate microscale biological features of human tongue surface, we studied the topological profiles of the two types of papillae (Figure 3) on the different silicone surfaces (see Table 2). The profiles correspond to single papillae of average dimensions for each surface presented in Figure 3. The measurements were performed in a line parallel to the direction from tip to back of the tongue, which will correspond to the direction of movement in our tribological tests assuming unidirectional motion.

In Figure 3a, the profile of fungiform papillae shows the monotonic dome-shape in the surfaces molded using the tongue mask (PDMStongue, Ecohbtongue and Ecohltongue). The profile of the dome shape-like bump (red dotted line) on the 3D-printed artificial surface (Ecohbprint) are close replicas of the fungiform papillae on surfaces PDMStongue, Ecohbtongue and Ecohltongue (Figure 3a). The unperturbed shape of filiform papillae is crown-like, having a cylindrical base with thin filaments on top of the base. However, due to the weight of the thin layer of the impression material (either polyvinyl siloxane or alginate), the thin filaments are expected to be compressed and imprinted as irregularities on top of the base of the filiform papillae. The profiles in Figure $3 \mathrm{~b}$ correspond to filiform papillae subjected to the weight of the impression material (PDMStongue), showing the cylindrical like shape of the base with a nonmonotonic shape at the topmost likely due to squashed filaments. Of more importance here is the profile corresponding to the $3 \mathrm{D}$-printed surface i.e. Ecohbprint (Figure $3 \mathrm{~b}$ ), which is modeled as a cylindrical shape (Figure $2 \mathrm{~d}$ ) is an excellent facsimile of the real human filiform papillae (Ecohbtongue) when made using the same polymer, i.e., Ecoflex 00-30.

Our findings demonstrate that we can successfully emulate real tongue surface in terms of concrete features varying in dimensions, shapes and densities using random digital designing, 3D-printing with high resolution, and replicamolding.

3.2. Collision Probability As a Measure of Mechanosensing. Presence of micropapillated features on tongue surfaces have been recently recognized to be vital to mechanosensing and textural perception. ${ }^{37}$ Nevertheless, whether or not the arrangements and density of papillae influence their mechanosensing efficiency remain poorly understood. In general, a regular square grid arrangement ${ }^{17,38}$ of papillae has been used as a basic model of papillae distribution in literature. However, as seen in Figure 1, the distribution of papillae is clearly complex and irregular. Therefore, it is crucial to ask whether the spatial Poisson point-based randomization of papillae used to fabricate the artificial 3D-printed and replica-molded tongue surfaces are closer to the papillae distribution in real tongue and more importantly simulate the mechano-reception performance requirement.

We introduce collision probability as the first ever conceptual approach to measure mechanosensing as a function of papillae arrangement. In a short movement of a particle with the linear trajectory over the tongue surface, the particle is more likely to encounter a direct collision with one or more papillae when the papillae are arranged more densely. Collision probability per unit distance of a papillae arrangement is defined as the probability that a food or fluid particle hits a papilla while traveling in a linear motion for a unit distance.

Here we study collision probability for papillae in square grid (Figure 4a) or Poisson distributions (Figure 4b). Our
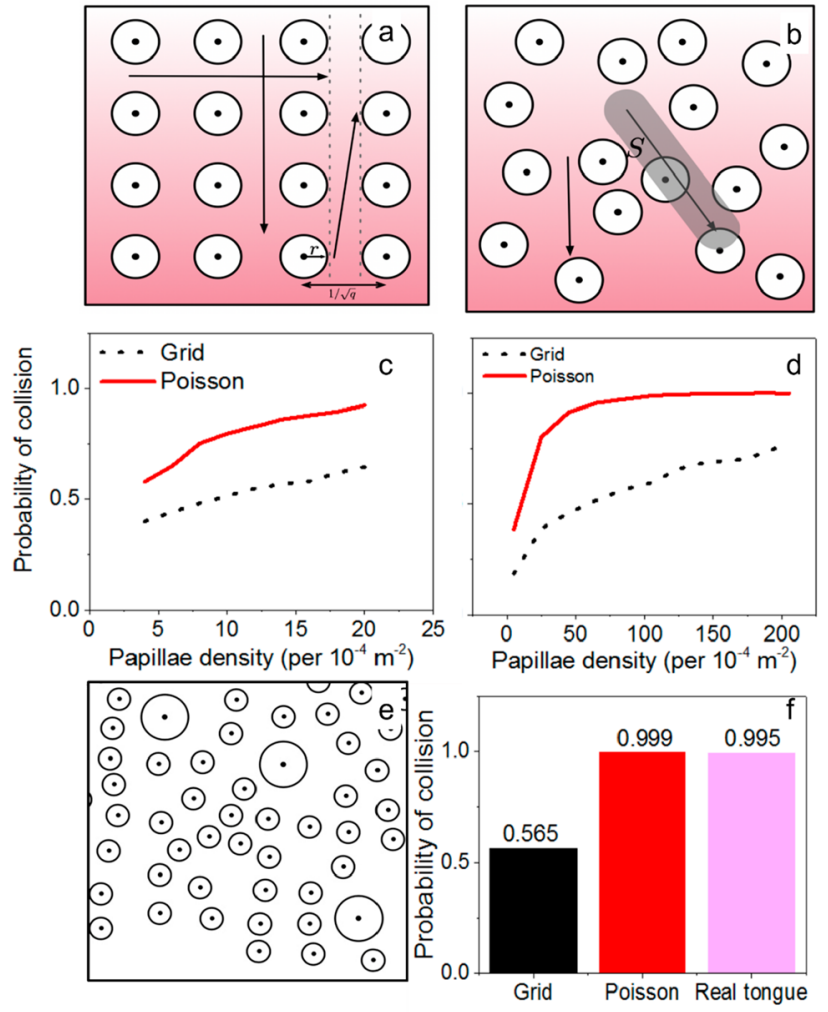

Figure 4. Modeling of collision probability per unit distance of papillae. Schematic illustration of collision probability of papillae distributed in a regular (a) square-grid surface and (b) randomized arrangement using Poisson point process with $r$ and $q$ being the radius and density of papillae derived from Table 1 (points within the circle show the center of the papillae). Computational simulation of probability of a particle hitting (c) fungiform papillae or a (d) filiform papillae in $1 \mathrm{~cm}$ linear distance in square grid or randomized arrangement. (e) Schematic illustration of both fungiform and filiform papillae based on real data of distribution in a real human tongue shown in Figure 1c with large circle representing fungiform and small circle representing filiform papillae and consequently computational simulation of (f) probability of a particle hitting the papillae showing close resemblance of randomized arrangement used in creating the artificial model in this study and a real human tongue. Simulations were performed on a 2D model of square grid, artificial randomized surface and real tongue surface with mean papillae diameter shown in Table 1. Each data point corresponds to 2000 runs of the program.

main theoretical result is that under a Poisson distribution (random) model, as the density of papillae per unit area increases, the collision probability per unit distance increases rapidly. In fact, the probability that a random displacement does not hit any papilla at all decreases exponentially fast with the density $q$ per unit area, as shown in the following theorem.

Theorem 1. When papillae are in a Poisson distribution with density $q$ per unit area, the collision probability per unit distance is at least $1-\mathrm{e}^{-2 r q}$.

Proof. Suppose the surface under consideration has area $U$, so that the number of papillae is $q U$. Let us represent by $S$, the region around the motion, such that any papilla centered in $S$ will be hit by the motion of length 1 (see Figure $4 b$ ). The area $S$ is $2 r \times 1+\pi r^{2}$.

The probability that any particular papilla center is in the region of size $S$ is given by $S / U$, and the probability of the papilla not being in the region is $1-S / U$. The probability that none of the papillae are in the area $S$ is $(1-S / U)^{q U}$. 
Therefore, the probability that one or more of the papillae are in the region $S$ is at least:

$$
\begin{aligned}
& \geq 1-(1-S / U)^{q U} \\
& \geq 1-(1-S / U)^{\frac{U}{S} S q} \\
& \geq 1-\left(1-\frac{1}{e}\right)^{S q} \geq 1-\mathrm{e}^{-2 r q}
\end{aligned}
$$

The final steps of the result follow from the well-known inequality $(1-1 / x)^{x} \leq 1 / e$.

When two different types of papillae (e.g., filiform and fungiform) with different radii $\left(r_{1}\right.$ and $\left.r_{2}\right)$ and different densities $q_{1}$ and $q_{2}$ are considered, the probability generalizes to $1-\mathrm{e}^{-2\left(r_{1} q_{1}+r_{2} q_{2}\right)}$, since the distributions are independent.

In the grid case (Figure $4 \mathrm{a}$ ), the collision probability increases more slowly. In a unit square, the linear separation between papillae is $\frac{1}{\sqrt{q}}$, leaving channels of width $\frac{1}{\sqrt{q}-2 r}$ between rows. Any displacement within the channel at an angle up to $\sin ^{-1}\left(\frac{1}{\sqrt{q}-2 r}\right)$ stays within the channel, and thus does not collide with any papilla. For $0<\theta \leq 1, \theta<\sin ^{-1} \theta$, thus a unit displacement at any angle up to $\left(\frac{1}{\sqrt{q}-2 r}\right)$ does not hit any papilla. Thus, the probability of a random unit displacement not hitting any papilla is at least $\frac{1}{2 \pi} \frac{1}{\sqrt{ } q-2 r}$, and collision probability is at most $1-\frac{1}{2 \pi} \frac{1}{\sqrt{ } q-2 r}$. Thus in a grid arrangement, the probability of not hitting any papilla decreases only as $\frac{1}{\sqrt{q}}$ : the square root of $q$, which is a much slower rate than the exponential rate for random distribution.

For simplicity of analysis, we have considered here movements of point particles. The analytic results carry over to larger particles of radii $s$ with a minor modification where in place of papillae radius $r$ we use the sum $(r+s)$, so that the bound in Theorem 1 becomes $1-\mathrm{e}^{-2(r+s) q}$.

We carried out simulations on artificially generated distributions of varying densities, and measured the probability of a displacement of $1 \mathrm{~cm}$ colliding with one or more papillae. Figure $4 c, d$ shows the results of these simulation trials. At each density value, 20 different arrangements were generated, and 100 random displacements of $1 \mathrm{~cm}$ were checked for collision with papillae.

Thus, each data point in Figure 4c,d corresponds to the results of 2000 trials. The main observation that at usual papillae densities corresponding to the human tongue shown in Table 1, the typical fungiform (Figure 4c) and filiform (Figure 4d) densities result in over $90 \%$ probability of collision and $99 \%$ probability respectively, in Poisson point processbased randomized arrangement. For similar densities, grid arrangement produces a collision probability of only about $60 \%$ (Figure $4 c, d$ ). We observe that in a grid arrangement, due to the linear alignment of papillae, there are clear horizontal and vertical channels between rows, where a motion does not face any obstruction.

Additionally, we recorded the positions of filiform and fungiform papillae on a section of a real human tongue (schematically illustrated in Figure 4e based on real position of the papillae in Figure 1c), and compared the collision-rate with those of artificial distributions created in this study (Figure 3a) containing both filiform and fungiform at their mean densities (Table 1). The result in Figure $4 \mathrm{f}$ shows that grid distribution produces a collision rate of only about $56 \%$, while the results of the real tongue surface and the Poisson distribution are almost identical at over $99 \%$. Thus, the results were consistent in both theoretical analysis and simulation experiments clearly giving a predictive impression that the artificial topology created using the Poisson point process closely resembles the mechanosensing performance of a real human tongue.

We note that papillae arrangement, and therefore collision probability, will also have a bearing on lubrication. However, the relation between these quantities is an intricate question that will require further study.

3.3. Emulating the Real Oral Contact Pressure. Besides topology, it is critical to consider other design requirements in the biomimicry process i.e. the deformability of the human tongue that profoundly influence the mechanical performance. Since we worked with human tongue masks in this study, the deformability of real human tongues could not be used as positive controls. Instead, pig tongues were used for the deformability study (Supplementary Figure S1a) and wettability measurement (Supplementary Figure S1b) and have Young's modulus of $<5.0 \mathrm{kPa}$ (Supplementary Figures S1a,c) irrespective of the positioning of the tongue, in line with previous reported values. ${ }^{39}$ The fungiform and filiform papillae of pig tongues (Supplementary Figure S1d,e) have similar shapes to their human counterparts (Figure 1d,e), but the papillae of pig tongues are about half the size of the human counterparts. Nevertheless, the viscoelastic properties of the pig tongue are comparable to those reported for in vivo human gingival surfaces. ${ }^{40,41}$

To create deformable soft tongue-like surfaces, different silicone surfaces were employed (Table 2), and simple compression tests were performed (Figure 5a). Uniquely, Ecoflex 00-30 surfaces with and without the addition of surfactant, demonstrated Young's modulus around 130.0 and $120.0 \mathrm{kPa}$, respectively, i.e., 1 order of magnitude lower modulus as compared with those of PDMS surfaces $(\sim 2.4$ $\mathrm{MPa}$ ), the latter considered as the current state-of-the art for oral tribological surfaces. ${ }^{7}$ Addition of Span 80 as a surfactant to these soft elastomers (Ecoflex 00-30) did not modify the mechanical properties significantly (Figure 5a). Considering the maximum pressures developed in the soft tongue-palate contact are around $50.0 \mathrm{kPa},{ }^{22,23}$ we expect the softer Ecoflex 00-30 to be a substantial improvement with respect to the current state-of-the-art, ${ }^{7}$ endowing a more realistic distribution of forces during mechanical contact. To confirm this improvement of using Ecoflex 00-30 over PDMS better, dry contact pressure is calculated for the $3 \mathrm{D}$-printed surfaces using Hertz theory (see eqs 4-8) in the method section for details).

In the case of Ecohbprint having a Young's modulus of 130.0 $\mathrm{kPa}$ (Figure 5a), the load $(1.0 \mathrm{~N})$ is distributed between the two types of papillae, with average values of pressure in the fungiform and filiform species being 33.0 and $9.8 \mathrm{kPa}$, respectively. The indentation of fungiform papillae is about $200.0 \mu \mathrm{m}$ leaving a gap between the top and bottom surfaces of $200.0 \mu \mathrm{m}$. Using PDMS surfaces with a Young's modulus of $2.4 \mathrm{MPa}$ (Figure 5a), the indentation is about $50 \mu \mathrm{m}$ with pressure distributed only on fungiform papillae having an average value of $384.0 \mathrm{kPa}$, i.e., 1 order of magnitude higher in comparison to Ecoflex 00-30 surfaces. In agreement with the pressure calculations, the lower Young's modulus of Ecoflex 00-30 approximate to real oral pressure $(\sim 50 \mathrm{kPa})$ allowing 

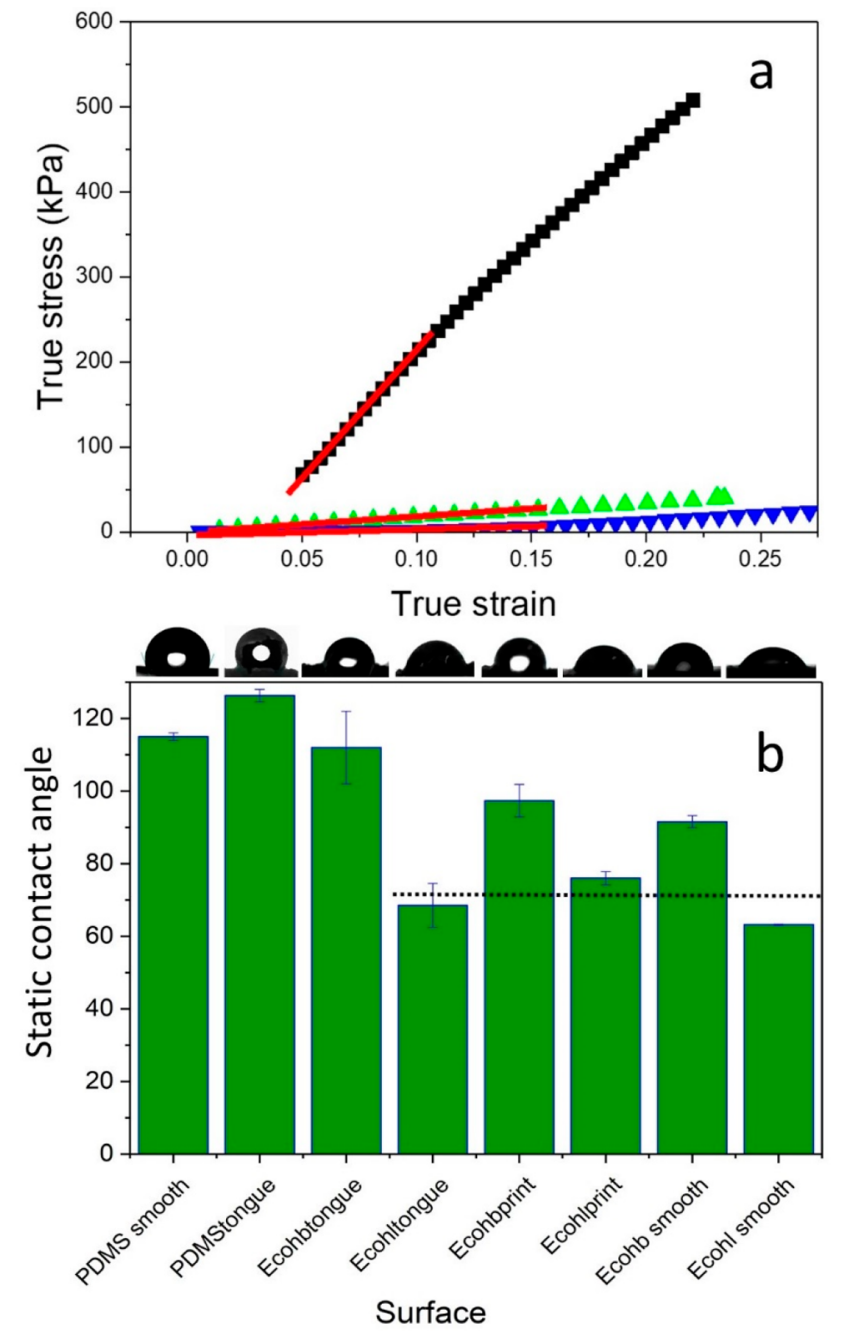

Figure 5. Comparison of material properties of tongue masks and 3Dprinted tongue-like polymeric surfaces. (a) Young's modulus of PDMS (black square, $E=2.4 \mathrm{MPa}$ ), Ecoflex 00-30 (green up triangle, $E=0.13 \mathrm{MPa}$ ) and Ecoflex 00-30 + Span 80 (0.5 wt \%) (blue down triangle, $E=0.12 \mathrm{MPa}$ ), latter used for fabricating the biomimetic tongue, i.e., Ecohlprint. Red solid lines represent fittings to the linear regime where the slope represents Young's modulus of the polymeric materials. (b) Wettability of the silicone surfaces created using tongue mask as well as 3D-printed micromolded master (see Table 2 for nomenclature). Visual images of the drop shape for each surfaces are shown on the top of their corresponding error bars, the latter representing the standard deviations. The black dashed line represent the wettability of real tongue surface using pig's tongue as a model (see Supplementary Figure S1b), the static contact angle value is within the range of real human gingival surfaces. ${ }^{40}$

the participation of both fungiform and filiform papillae like asperities in the mechanical contact. These calculations will be further used to discuss the friction curves obtained for the different surfaces in the tribological performance section of artificial and real tongue masks.

3.4. Wettability Analogous to That of a Pig's Tongue. There is no doubt that combining architecture with surface chemistry is a crucial step for designing biorelevant tribological surfaces. To date, no textured tribological surfaces have emulated the wettability of a human tongue. ${ }^{7}$ Figure $5 \mathrm{~b}$ shows the static contact angle of a water drop deposited on the surface, for PDMS, Ecoflex 00-30 and Ecoflex 00-30 with surfactant (Span 80) prepared using variety of surfaces prepared in Table 2. Smooth PDMS $\left(115.0^{\circ} \pm 1.0^{\circ}\right)$ and Ecoflex 00-30 $\left(92.0^{\circ} \pm 2.0^{\circ}\right)$ surfaces (with no topological features) are both hydrophobic in nature. Interestingly, contact angle on surfaces made of PDMS and Ecoflex 00-30 increases when texture is introduced, put simply, roughness is modified by the presence of fungiform and filiform papillae using either a tongue mask or the 3D-printed artificially created master (PDMStongue, $126.0^{\circ} \pm 2.0^{\circ}$ and Ecohbtongue, $112.0 \pm$ $10.0^{\circ}$ ). Similarly, Ecoflex 00-30 surfaces obtained using 3D printing techniques (Ecohbprint) had a slightly larger contact angle $\left(98.0^{\circ} \pm 4.0^{\circ}\right)$ in comparison to the smooth Ecoflex 0030 surface $\left(92.0^{\circ} \pm 2.0^{\circ}\right)$. Similar increases in the static contact angle have been reported before on surfaces containing asperities with heights having a Gaussian distribution. ${ }^{42}$

Herein, we worked with pig tongues as controls which have a static water contact angle of $\sim 77^{\circ}$ (Supplementary Figure S1b) and later corroborates the wettability values reported for in vivo human gingival surfaces. ${ }^{40,41}$ In order to obtain the hydrophilicity similar to that of the pig's tongue surface, wettability modification of Ecoflex 00-30 was obtained by addition of a surfactant, i.e., Span $80^{29}$ (Figure $5 \mathrm{~b}$ ). Surfaces Ecohl smooth (Ecoflex 00-30 modified by 0.5 wt \% Span 80 ) has a contact angle of $63.0^{\circ} \pm 0.2^{\circ}$, considerably lower in comparison to that of nonmodified Ecoflex 00-30 (Ecohb smooth). Textured surfaces containing Span 80, i.e., Ecohltongue and Ecohlprint, have relatively low values of contact angle of $69.0^{\circ} \pm 6.05^{\circ}$ and $76.0^{\circ} \pm 2.0^{\circ}$ respectively. Thus, modification of Ecoflex 00-30 with the addition of $0.5 \mathrm{wt}$ $\%$ Span 80 creates surfaces with wettability close to that of tongue surface $\left(\sim 77^{\circ}\right.$, shown in dashed line in Figure $\left.4 \mathrm{~b}\right)$.

Supplementary Figure S2a,b shows scanning electron microscopy images of surfaces Ecohltongue (S2a) and Ecohlprint (S2b). Both surfaces show random distribution of fungiform and filiform papillae like shapes. The surface designed for $3 \mathrm{D}$ printing (Ecohlprint) is observed as a relatively smooth plane containing the protuberances shaped as papillae, while surface Ecohltongue shows a relatively rough space among papillae. Despite these differences, we hypothesize that larger sized features (papillae) are expected to dominate the tribological performance of the surfaces.

Taken together these experimental results and computational simulations, we demonstrate that Ecohlprint represents the biomimetic tongue surface duplicating the texture and wettability of the human tongue surface. Although the Young's modulus of Ecohlprint is still an 1 order of magnitude larger than the real tongue tissue (Figure 5a), Ecohlprint being 1 order of magnitude softer than the current standard (PDMS) surfaces is clearly advantageous and more importantly endows optimized (calculated) dry contact pressure to closely model the mechanical interaction between the tongue and products undergoing oral processing.

3.5. Mimicking the Tribological Performance of Real Human Tongue Surface. Following the biomimicry of the topographical features and wetting properties, these last experiments (Figures 6 and 7) demonstrate the relevance of these biomimetic soft tongue-like to tribological applications. Here, we demonstrate unique capability of these biomimetic surfaces to emulate the tribological response of the tongue masks during oral processing particularly in the case of hydrophilic surfaces. The tribological performance of all surfaces was tested in a rotational rheometer working in normal force control mode (see schematic diagram in Figure 


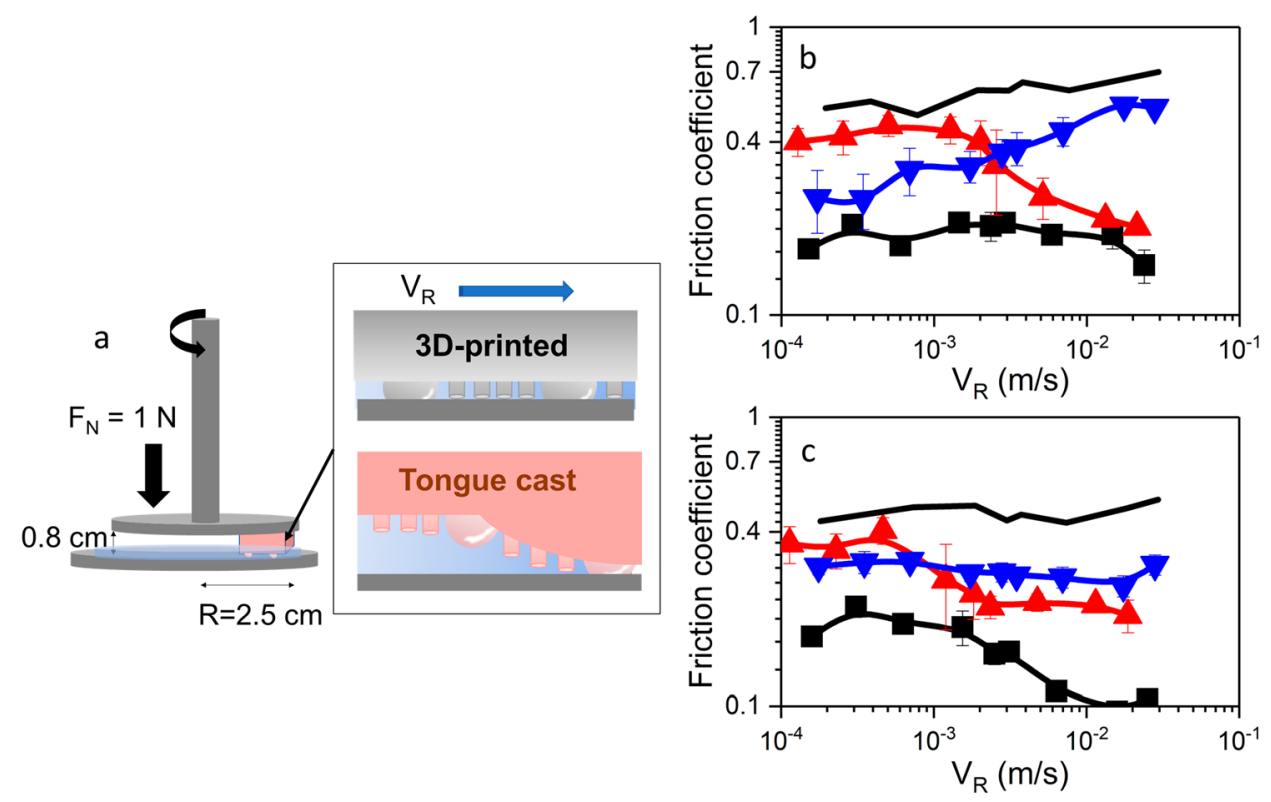

Figure 6. Tribological performance of hydrophobic tongue masks and hydrophobic 3D-printed tongue-like polymeric surfaces. (a) Schematic representation of the tribological setup adapting a rotational rheometer. The friction coefficient as a function of linear speed $\left(\mathrm{V}_{\mathrm{R}}\right)$ for hydrophobic silicone surfaces lubricated with model hydrophilic lubricants for, (b) $1.0 \mathrm{wt} \%$ xanthan gum solutions, and (c) 10.0 wt \% whey protein solutions. Surfaces used were PDMStongue (black squares), Ecohbtongue (red up triangle) and Ecohbprint (blue down triangle). Continuous black lines represent the lubrication performance of the respective fluids on smooth PDMS surfaces without any topographic features.
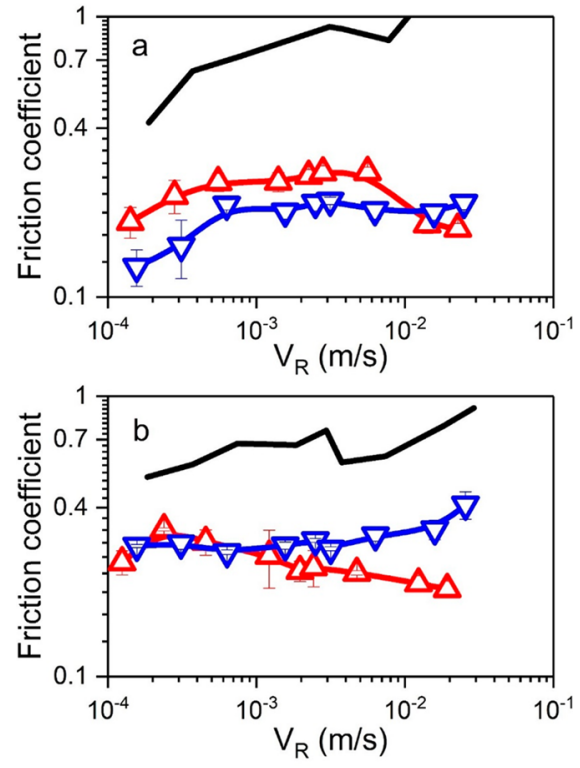

Figure 7. Tribological performance of hydrophilic tongue masks and hydrophilic 3D-printed biomimetic tongue-like polymeric surfaces. The friction coefficient as a function of linear speed $\left(V_{R}\right)$ for hydrophilic silicone surfaces lubricated with model hydrophilic lubricants for, (a) $1.0 \mathrm{wt} \%$ xanthan gum solutions and (b) $10.0 \mathrm{wt}$ $\%$ whey protein solutions. Surfaces used were Ecohltongue (red up triangle) and Ecohlprint (blue down triangle). Continuous black lines represent the lubrication performance of the respective fluids on smooth PDMS surfaces without any topographic features.

$6 a$ and eq 3 in the method section for calculation of friction coefficients).

To test the mechanical performance of the surfaces, two model food fluids, i.e., whey protein solution and xanthan gum solution were used as aqueous lubricants to demonstrate the performance of the surfaces under load and speed conditions relevant for oral lubrication studies.

Xanthan gum is a shear thinning fluid with significantly higher viscosity in comparison to the whey protein solution in the range of shear rates measured (Supplementary Figure S3a). The whey protein solution shows Newtonian-like behavior at the relevant shear rates. Given the significantly larger viscosity of xanthan gum in comparison to the whey protein solution, from a hydrodynamic point of view, it is expected that xanthan gum decrease friction to a larger extent. ${ }^{21}$ However, the lubrication performance of these fluids were very similar when tested in the current state-of-the-art device, i.e., smooth PDMS ball-on-disk tribological setup working under common oral mimicking conditions showing overlapping friction coefficient curves (Supplementary Figure S3b), suggesting that whey protein lubrication is related to nonhydrodynamic forces, with a likely mechanism being hydration lubrication. ${ }^{43}$ Noteworthy that the experimental conditions on the ball on disk tribometer are different in comparison to those used with the tongue mimicking surfaces in this work. The total friction on the ball on plate contact is the product of both rolling and sliding friction, though the latter is commonly significantly larger. Thus, the difference in pressure and absolute values of contact area are expected to differ significantly between the two setups. Since both types of mechanical contacts are considered as soft and working under low pressure having no influence on the physical properties of the test fluids, we expect that the possible difference in the functional form of the friction curves (as function of speed) between the two setups is more sensitive to topography, Young modulus, and wettability of the surfaces.

To confirm that the newly designed biomimetic surfaces are analogous to replica molded surfaces of real tongue masks, first, we compare the tribological behavior of the hydrophobic surfaces. i.e.. replica molded tongue mask (Ecohbtongue), 3Dprinted replica-molded hydrophobic surface (Ecohbprint) and 
a less deformable version of the tongue surface (PDMStongue; Figure $6 \mathrm{~b}, \mathrm{c})$. Following that, we shift our focus to hydrophilic surfaces (Ecohltongue, Ecohlprint; Figure 7a,b).

Figure $6 \mathrm{~b}$ shows the friction coefficient curves as function of linear speeds $\left(V_{R}\right)$ for the hydrophobic surfaces in the presence of 1.0 wt \% xanthan gum solution. Noteworthy, that images of the $3 \mathrm{D}$ surfaces obtained by surface reconstructions of the optical scans before and after being used in tribological experiments (Supplementary Figure S4) reveal that neither of these three aforementioned surfaces showed any damage due to the stress imposed during tribological tests. The less deformable PDMStongue shows the friction coefficient independent of speed with values around 0.2 (Figure $6 \mathrm{~b}$ ) relatively lower in comparison to the other surfaces due to the lower effective contact area (less deformable surface).

In an ideal case scenario of perfect biomimicry, Ecohbtongue and Ecohbprint should have shown the same friction coefficient curves but this is not the case (Figure $6 \mathrm{~b}$ ). The friction coefficient measured with softer Ecohbtongue increases slightly at the lowest speed up to a speed of $2.5 \times 10^{-3} \mathrm{~m} / \mathrm{s}$ reaching a friction coefficient value of 0.4 . On increasing the speed, the friction coefficient decreased monotonically reaching a value around 0.2 at the highest speed of $2.5 \times 10^{-2} \mathrm{~m} / \mathrm{s}$. It is worth noting that the human tongue works as a "system" where by surface mechanical properties, roughness, texture and form interact with lubricant properties to contribute to the effectiveness of the overall lubrication.

The decrease in friction coefficients on increasing speed found for Ecohbtongue (1.0 wt \% xanthan gum and $10.0 \mathrm{wt} \%$ whey protein solutions) and PDMStongue (10.0 wt \% whey protein solutions) can be related to the underlying shape inherited from the tongue mask instead of the surface texture (papillae distribution) and rheological properties of the fluid. This is shown schematically in Figure 6a showing the underlying shape obtained from the $3 \mathrm{D}$ optical scan as described in the method section. For simplification we have chosen a $1 \mathrm{D}$ example where the profile corresponds to a line in the direction of displacement. This hypothesis is further corroborated by PDMStongue $1.0 \mathrm{wt} \%$ xanthan gum solutions where the coefficient of friction was seen to be monotonic with respect to increasing entrainment velocities; likely due to the increased moduli of PDMS hindering the formation of any loading bearing lubricating film.

Our hypothesis is that the underlying shape of tongue cast models contributes to pressurize the fluid within the interface, lowering the friction by decreasing the total load supported by direct contact between the surfaces. Increasing speed would increase the pressure within any lubricating film, thereby decreasing further the contact friction. Similar observations have been made computationally by Fowell et al. (2006) ${ }^{44}$ who found that the inlet geometry, in particular its convergence, plays a major role in fluid load support and friction reduction and enhanced lubricant film formation and reduced friction of textured bearings. Within lubrication science, it is common to use the lambda ratio $\left(\lambda=\frac{\text { elastohydrodynamic film thickness }}{\text { composite surface roughness }}\right)$ to describe the lubrication mechanisms occurring at contacting surfaces. However, the limitations associated with application of this model to "soft" tribological systems are well documented and application of this analysis method to our system would suggest a purely boundary friction mechanism owing to the high surface roughness. ${ }^{45}$ It is further hypothesized that the surface roughness of these systems will likely contribute the promotion of soft micro-EHL whereby local pressure generation will be perturbed by the flattening of the surface profile. A much bigger "real" lambda ratio will be promoted at the interface and as such normal load can be part supported by localized fluid pressurization within the contact.

Friction coefficients obtained for Ecohbprint increase monotonically with speed in the whole experimental window (Figure $6 \mathrm{~b}$ ). At the lowest speed of $1.5 \times 10^{-4} \mathrm{~m} / \mathrm{s}$, the friction coefficient was about 0.3 , increasing to a value of 0.5 at the highest speed of $2.5 \times 10^{-2} \mathrm{~m} / \mathrm{s}$. Based on papillae compression calculations, the friction force measured is a combination of the surface-to-surface contact and the shear stress generated in the fluid trapped between surfaces. This is also confirmed by the absence of speed dependence with the smooth surfaces (black lines), which are not as capable to trap fluid as the textured surfaces. The underlying flat shape of the printed surfaces (Figure 6a) and parallel nature of the contact is less susceptible to fluid pressurization and fluid load support within the interface and thus no decrease in friction with speed is observed. This again suggests that the difference in tribological behavior between the real tongue mask and the 3D-printed surfaces (Figure 6b) is largely attributed to the underlying shape of the real tongue as discussed above.

Figure $6 c$ shows the lubrication performance of the same hydrophobic surfaces when the surfaces are in the presence of whey protein solution (10.0 wt $\%$ protein content). The friction coefficient obtained for PDMStongue lubricated with the whey protein solution oscillates around a value of 0.2 for speeds ranging from $1.5 \times 10^{-4}$ to $2.5 \times 10^{-3} \mathrm{~m} / \mathrm{s}$. At higher speeds, the friction coefficient decreases reaching a value of 0.1 at the speed of $2.5 \times 10^{-2} \mathrm{~m} / \mathrm{s}$. Apart from the absolute values of the friction coefficient, the speed dependence displayed by the curves show a significant difference due to Young's modulus for surfaces PDMStongue and Ecohbtongue, causing differences in pressure distribution in the contact. Of more importance here is that the friction curves obtained for Ecohbtongue and Ecohbprint are similar, with the friction coefficient showing no significant dependence on speed with values around 0.3 , suggesting that a nonhydrodynamic force dominates the frictional behavior circumventing the shape factor difference between the two surfaces, as discussed before. This also indicates that biomimicking both the texturedarchitecture and surface chemistry of soft deformable surface is crucial to achieve the replication of tribological performance.

It is important to remark that unlike the popular PDMS ball on disk setup, the experimental design using the surface PDMStongue is capable of distinguishing between the two test fluids due to the inherited complex tongue topography in the later. Surprisingly, despite the lower viscosity and relatively lower hydrated mass than xanthan gum ${ }^{33}$ under quiescent conditions as measured using QCM-D (Supplementary Figure S5), whey protein is capable of decreasing friction coefficient to a larger extent in the case of PDMStongue. This indicates that other techniques are necessary to establish relationships between hydration and adsorption in nonquiescent conditions, however this is out of the scope of the present work.

In order to understand the role of wettability on the tribological performance of the tongue-mimicking surface, Figure 7 shows the lubrication performance of surfaces made of the hydrophilic silicone material Ecohltongue and Ecohlprint, latter being the biomimetic tongue surface. Figure 7 a shows the friction curves for surfaces lubricated by the xanthan gum solution. Curves obtained for both tongue mask 
and biomimetic surfaces have similar shape up to a speed of $0.01 \mathrm{~m} / \mathrm{s}$ with the friction coefficients obtained for Ecohltongue being slightly lower in comparison to Ecohlprint.

As one might expect, the absolute values of friction obtained for the hydrophilic surfaces (Figure 7a) are lower in comparison to the hydrophobic surfaces (Figure $6 \mathrm{~b}$ ) due to the enhanced aqueous lubrication associated with surface wettability. ${ }^{46}$ Above a speed of $0.01 \mathrm{~m} / \mathrm{s}$, the friction coefficient obtained for Ecohltongue (Figure 7a) decreases with speed at values similar to its hydrophobic counterpart (Figure 6b) owing to the surface curvature, as explained before. It is clear that increasing wettability of the surfaces in the biomimicry process improves the aqueous lubrication, screening hydrodynamic forces in the fluid film and thus the shape factor tends to play a less important role as compared to that observed in hydrophobic surfaces.

Figure $7 \mathrm{~b}$ shows the friction curves for the hydrophilic surfaces, i.e., Ecohltongue and Ecohlprint lubricated with whey protein solution. Friction curves for both surfaces overlap for speeds below $0.01 \mathrm{~m} / \mathrm{s}$ with a constant value of about 0.3 . Above $0.01 \mathrm{~m} / \mathrm{s}$, the friction coefficient increases slightly only in the case of Ecohlprint. The combination of wettability and texture in Ecohlprint mimicking the tongue tissue is capable of distinguishing between the two fluids employed here as lubricants, which was not possible using the standard setup for oral tribology studies (Supplementary Figure S3b).

Therefore, these unique frictional performance results analogous to those of replica-molded tongue masks demonstrate clearly the tribological application of this newly designed 3D tongue-like biomimetic surfaces with accurate quantification.

\section{CONCLUSIONS}

A 3D-printed surface was designed in this study, to mimic topological features, roughness as well as mechanical (elasticity) and chemical (wettability) properties of an average human tongue with the aim of accurately measuring oral tribological properties. Using 3D printing and soft lithography techniques, we designed this biomimetic surface containing fungiform and filiform papillae-like asperities for the first time that are randomly distributed. Experimental results of biomimicry show that the surface having Young's modulus about $100.0 \mathrm{kPa}$, a water/air/solid contact angle around $76^{\circ}$ closely resembles the natural anatomical architecture of the human tongue surface. We define collision probability as a novel theoretical measure, excellently matched by computational simulations, to quantify mechanosensing of different papillae arrangements. These computational simulations reveal that the randomness of the features rendered in this newly fabricated surface provide mechanosensing similar to that of a real tongue surface. Finally, friction test results performed in pure sliding conditions and low pressures (below $10.0 \mathrm{kPa}$ ) in this model 3D biomimetic tongue-like surface reveal a similar performance with a mask using the same polymeric material of a natural human tongue surface. This innovative model surface is expected to enable mechanical testing under oral tribological shear of food, orally administered drugs and oral care products providing proximity of the oral tribological surface to real biological tissue. The oral tribological testing with this sophisticated tongue-like surface will set the precedence for identifying fundamental oral lubrication mechanisms and consequently enable addressing basic mechanobiological questions. The biomimicry based on intrinsic material properties and biological randomness as well as functional emulation makes the $3 \mathrm{D}$ tongue-like surface a promising basis for developing an advanced, sensitive screening platform to accelerate the development cycle of nutritional, biomedical and clinical applications, where oral lubrication performance is a key requirement. In addition, the biomimetic approaches used in this study starting from human tongue to an in vitro tribological setup might find application in the field of soft robotics, where there is a huge interests in biomimetic and bioinspired systems.

\section{ASSOCIATED CONTENT}

\section{SI Supporting Information}

The Supporting Information is available free of charge at https://pubs.acs.org/doi/10.1021/acsami.0c12925.

Topographic analysis of selected areas of human tongue surface; material characteristics of pig tongue; electron micrographs of soft hydrophilic polymeric tongue and 3D-printed tongue-like surfaces; rheology and tribology of model hydrophilic lubricants; positive $3 \mathrm{D}$ optical scan and surface reconstruction of polymeric surfaces before and after tribological testing and adsorption behavior of whey protein and xanthan gum on PDMS surfaces (PDF)

\section{AUTHOR INFORMATION}

\section{Corresponding Author}

Anwesha Sarkar - Food Colloids and Bioprocessing Group, School of Food Science and Nutrition, University of Leeds, Leeds LS2 9JT, United Kingdom; 다이.org/0000-0003-17422122; Email: A.Sarkar@leeds.ac.uk

\section{Authors}

Efren Andablo-Reyes - Food Colloids and Bioprocessing Group, School of Food Science and Nutrition, University of Leeds, Leeds LS2 9JT, United Kingdom

Michael Bryant - Institute of Functional Surfaces, School of Mechanical Engineering, University of Leeds, Leeds LS2 9JT, United Kingdom

Anne Neville - Institute of Functional Surfaces, School of Mechanical Engineering, University of Leeds, Leeds LS2 9JT, United Kingdom

Paul Hyde - School of Dentistry, University of Leeds, Leeds LS2 9JT, United Kingdom

Rik Sarkar - School of Informatics, University of Edinburgh, Edinburgh EH8 9AB, United Kingdom

Mathew Francis - Food Colloids and Bioprocessing Group, School of Food Science and Nutrition, University of Leeds, Leeds LS2 9JT, United Kingdom

Complete contact information is available at: https://pubs.acs.org/10.1021/acsami.0c12925

\section{Author Contributions}

A.S. designed the research question and supervised the project. E.A.R., M.B., A.N., and A.S. designed the experimental protocol and contributed to the data analysis of the experimental results. E.A.R. performed optical measurements, surface generation, scanning electron microscopy of silicon surfaces, tribological and rheological experiments, and pressure calculations. P.H. performed experiments of collecting tongue impressions of the human participants. R.S. performed surface reconstruction and performed analysis and calculations related 
to the random nature of the surfaces. M.F. prepared some of the 3D-printed surfaces. E.A.R. and A.S. had primary responsibility for the final content; and all authors read, edited, and approved the final manuscript.

\section{Funding}

This project has received funding from the European Research Council (ERC) under the European Union's Horizon 2020 research and innovation program (Grant Agreement No. 757993).

\section{Notes}

The authors declare no competing financial interest.

The data presented in this article will be openly available from the University of Leeds Data Repository: https://doi.org/10. $5518 / 917$.

\section{ACKNOWLEDGMENTS}

The authors would like to thank Camille Hammersley (School of Mechanical Engineering, Faculty of Engineering and Physical Sciences, University of Leeds) for her technical support in using the Alicona InfiniteFocus instrument. Stuart Micklethwaite (LEMAS, Leeds Electron Microscopy and Spectroscopy Centre, Faculty of Engineering, University of Leeds) is acknowledged for his technical support in electron microscopy. Dr. Evangelos Liamas (School of Food Science and Nutrition, Faculty of Environment, University of Leeds) is acknowledged for his technical support in QCM-D experiments.

\section{ADDITIONAL NOTE}

${ }^{a}$ https://www.meshlab.net/

\section{REFERENCES}

(1) Noel, A. C.; Hu, D. L. Cats Use Hollow Papillae to Wick Saliva into Fur. Proc. Natl. Acad. Sci. U. S. A. 2018, 115 (49), 12377-12382.

(2) Harper, C. J.; Swartz, S. M.; Brainerd, E. L. Specialized Bat Tongue is a Hemodynamic Nectar Mop. Proc. Natl. Acad. Sci. U. S. A. 2013, 110 (22), 8852-8857.

(3) Chen, J.; Stokes, J. R. Rheology and tribology: Two Distinctive Regimes of Food Texture Sensation. Trends Food Sci. Technol. 2012, 25, 4-12.

(4) Stokes, J. R.; Boehm, M. W.; Baier, S. K. Oral Processing, Texture and Mouthfeel: From Rheology to Tribology and Beyond. Curr. Opin. Colloid Interface Sci. 2013, 18 (4), 349-359.

(5) Green, J. R.; Wang, Y. T. Tongue-Surface Movement Patterns during Speech and Swallowing. J. Acoust. Soc. Am. 2003, 113 (5), 2820-2833.

(6) Kullaamikkonen, A.; Sorvari, T. E. A Scanning ElectronMicroscopic Study of the Dorsal Surface of the Human Tongue. Cells Tissues Organs 1985, 123 (2), 114-120.

(7) Sarkar, A.; Andablo-Reyes, E.; Bryant, M.; Dowson, D.; Neville, A. Lubrication of Soft Oral Surfaces. Curr. Opin. Colloid Interface Sci. 2019, 39, 61-75.

(8) Sarkar, A.; Krop, E. M. Marrying Oral Tribology to Sensory Perception: A Systematic Review. Curr. Opin Food Sci. 2019, 27, 6473.

(9) Batchelor, H.; Venables, R.; Marriott, J.; Mills, T. The Application of Tribology in Assessing Texture Perception of Oral Liquid Medicines. Int. J. Pharm. 2015, 479 (2), 277-281.

(10) Cai, H.; Li, Y.; Chen, J. Rheology and Tribology Study of the Sensory Perception of Oral Care Products. Biotribology 2017, 10, 1725.

(11) Xu, F.; Liamas, E.; Bryant, M.; Adedeji, A. F.; Andablo-Reyes, E.; Castronovo, M.; Ettelaie, R.; Charpentier, T. V. J.; Sarkar, A. A Self-Assembled Binary Protein Model Explains High-Performance
Salivary Lubrication from Macro to Nanoscale. Adv. Mater. Interfaces 2020, 7, 1901549.

(12) Ma, S.; Lee, H.; Liang, Y.; Zhou, F. Astringent Mouthfeel as a Consequence of Lubrication Failure. Angew. Chem., Int. Ed. 2016, 55 (19), 5793-5797.

(13) Sánchez-Alcañiz, J. A.; Zappia, G.; Marion-Poll, F.; Benton, R. A Mechanosensory Receptor Required for Food Texture Detection in Drosophila. Nat. Commun. 2017, 8 (1), 14192.

(14) Zhang, Y. V.; Aikin, T. J.; Li, Z.; Montell, C. The Basis of Food Texture Sensation in Drosophila. Neuron 2016, 91 (4), 863-877.

(15) Pradal, C.; Stokes, J. R. Oral Tribology: Bridging the Gap between Physical Measurements and Sensory experience. Curr. Opin Food Sci. 2016, 9, 34-41.

(16) Wang, X. Q.; Wang, X. M.; Upadhyay, R.; Chen, J. S. Topographic Study of Human Tongue in Relation to Oral Tribology. Food Hydrocolloids 2019, 95, 116-121.

(17) Ranc, H.; Servais, C.; Chauvy, P. F.; Debaud, S.; Mischler, S. Effect of Surface Structure on Frictional Behaviour of a Tongue/ Palate Tribological System. Tribol. Int. 2006, 39 (12), 1518-1526.

(18) Carpenter, G.; Bozorgi, S.; Vladescu, S.; Forte, A. E.; Myant, C.; Potineni, R. V.; Reddyhoff, T.; Baier, S. K. A Study of Saliva Lubrication Using a Compliant Oral Mimic. Food Hydrocolloids 2019, 92, 10-18.

(19) Tsui, S.; Tandy, J.; Myant, C.; Masen, M.; Cann, P. M. Friction Measurements with Yoghurt in a Simulated Tongue-Palate Contact. Biotribology 2016, 8, 1-11.

(20) Torres, O.; Andablo-Reyes, E.; Murray, B. S.; Sarkar, A. Emulsion Microgel Particles as High-Performance Bio-Lubricants. ACS Appl. Mater. Interfaces 2018, 10 (32), 26893-26905.

(21) Andablo-Reyes, E.; Yerani, D.; Fu, M.; Liamas, E.; Connell, S.; Torres, O.; Sarkar, A. Microgels as Viscosity Modifiers Influence Lubrication Performance of Continuum. Soft Matter 2019, 15 (47), 9614-9624.

(22) Alsanei, W. A.; Chen, J. S. Studies of the Oral Capabilities in Relation to Bolus Manipulations and the Ease of Initiating Bolus Flow. J. Texture Stud. 2014, 45 (1), 1-12.

(23) Laguna, L.; Barrowclough, R. A.; Chen, J. S.; Sarkar, A. New Approach to Food Difficulty Perception: Food Structure, Food Oral Processing and Individual's Physical Strength. J. Texture Stud. 2016, 47 (5), 413-422.

(24) Liu, Y.; Hu, J.; Zhong, M.; Xu, W. A Novel, Simple and Rapid Method for the Detection of Melamine from Milk Based on Tribology Measurements. Tribol. Int. 2018, 119, 66-72.

(25) Kazhdan, M.; Hoppe, H. Screened Poisson Surface Reconstruction. Acm T Graphic 2013, 32 (3), 1.

(26) Merchan-Perez, A.; Rodriguez, J. R.; Gonzalez, S.; Robles, V.; DeFelipe, J.; Larranaga, P.; Bielza, C. Three-Dimensional Spatial Distribution of Synapses in the Neocortex: A Dual-Beam Electron Microscopy Study. Cereb Cortex 2014, 24 (6), 1579-1588.

(27) Truby, R. L.; Lewis, J. A. Printing Soft Matter in Three Dimensions. Nature 2016, 540 (7633), 371-378.

(28) Tumbleston, J. R.; Shirvanyants, D.; Ermoshkin, N.; Janusziewicz, R.; Johnson, A. R.; Kelly, D.; Chen, K.; Pinschmidt, R.; Rolland, J. P.; Ermoshkin, A.; Samulski, E. T.; DeSimone, J. M. Continuous Liquid Interface Production of 3D Objects. Science 2015, 347 (6228), 1349-1352.

(29) Marconati, M.; Pani, S.; Engmann, J.; Burbidge, A.; Ramaioli, M., A Soft Robotic Tongue to Manage Swallowing Disorders, Submitted. 2020

(30) Kazhdan, M.; Bolitho, M.; Hoppe, H. Poisson Surface Reconstruction. Proc. Symp. Geometry Processing 2006, 61-70.

(31) Rodahl, M.; Höök, F.; Krozer, A.; Brzezinski, P.; Kasemo, B. Quartz Crystal Microbalance Setup for Frequency and Q-Factor Measurements in Gaseous and Liquid Environments. Rev. Sci. Instrum. 1995, 66 (7), 3924-3930.

(32) Glumac, M.; Ritzoulis, C.; Chen, J. Surface Properties of Adsorbed Salivary Components at a Solid Hydrophobic Surface Using a Quartz Crystal Microbalance with Dissipation (QCM-D). Food Hydrocolloids 2019, 97, 105195. 
(33) Stokes, J. R.; Macakova, L.; Chojnicka-Paszun, A.; de Kruif, C. G.; de Jongh, H. H. J. Lubrication, Adsorption, and Rheology of Aqueous Polysaccharide Solutions. Langmuir 2011, 27 (7), 34743484.

(34) Macakova, L.; Yakubov, G. E.; Plunkett, M. A.; Stokes, J. R. Influence of Ionic Strength Changes on the Structure of Pre-Adsorbed Salivary Films. A Response of a Natural Multi-Component Layer. Colloids Surf., B 2010, 77 (1), 31-39.

(35) Tatara, Y. On Compression of Rubber Elastic Sphere over a Large Range of Displacements 0.1. Theoretical-Study. J. Eng. Mater. Technol. 1991, 113 (3), 285-291.

(36) Macosko, C. Rheology: Principles, Measurements, and Applications; Wiley-VCH, 1995.

(37) Lauga, E.; Pipe, C. J.; Le Révérend, B. Sensing in the Mouth: A Model for Filiform Papillae as Strain Amplifiers. Front. Phys. 2016, 4 (35), 35 DOI: 10.3389/fphy.2016.00035.

(38) Thomazo, J.-B.; Contreras Pastenes, J.; Pipe, C. J.; Le Reverend, B.; Wandersman, E.; Prevost, A. M. Probing In-Mouth Texture Perception with a Biomimetic Tongue. J. R. Soc., Interface 2019, 16 (159), 20190362.

(39) Ranc, H.; Elkhyat, A.; Servais, C.; Mac-Mary, S.; Launay, B.; Humbert, P. Friction Coefficient and Wettability of Oral Mucosal Tissue: Changes Induced by a Salivary Layer. Colloids Surf., A 2006, $276(1-3), 155-161$.

(40) van der Mei, H. C.; White, D. J.; Busscher, H. J. On the Wettability of Soft Tissues in the Human Oral Cavity. Arch. Oral Biol. 2004, 49 (8), 671-673.

(41) Cheng, S.; Gandevia, S. C.; Green, M.; Sinkus, R.; Bilston, L. E. Viscoelastic Properties of the Tongue and Soft Palate using MR Elastography. J. Biomech 2011, 44 (3), 450-454.

(42) Hu, S. T.; Cao, X. B.; Reddyhoff, T.; Puhan, D.; Huang, W. F.; Shi, X.; Peng, Z. K.; Dini, D. Three-Dimensional Printed Surfaces Inspired by Bi-Gaussian Stratified Plateaus. ACS Appl. Mater. Interfaces 2019, 11 (22), 20528-20534.

(43) Jahn, S.; Klein, J. Hydration Lubrication: The Macromolecular Domain. Macromolecules 2015, 48 (15), 5059-5075.

(44) Fowell, M.; Olver, A. V.; Gosman, A. D.; Spikes, H. A.; Pegg, I. Entrainment and Inlet Suction: Two Mechanisms of Hydrodynamic Lubrication in Textured Bearings. J. Tribol. 2007, 129 (2), 336-347.

(45) Dowson, D. Elastohydrodynamic and Micro-Elastohydrodynamic Lubrication. Wear 1995, 190 (2), 125-138.

(46) Bongaerts, J. H. H.; Fourtouni, K.; Stokes, J. R. Soft-Tribology: Lubrication in a Compliant PDMS-PDMS Contact. Tribol. Int. 2007, 40 (10-12), 1531-1542. 